\title{
ON MULTIPLE FREQUENCY POWER DENSITY MEASUREMENTS
}

\author{
GIOVANNI S. ALBERTI
}

\begin{abstract}
We shall give a priori conditions on the illuminations $\varphi_{i}$ such that the solutions to the Helmholtz equation

$$
\left\{\begin{array}{l}
-\operatorname{div}\left(a \nabla u^{i}\right)-k q u^{i}=0 \quad \text { in } \Omega \\
u^{i}=\varphi_{i} \quad \text { on } \partial \Omega
\end{array}\right.
$$

and their gradients satisfy certain non-zero and linear independence properties inside the domain $\Omega$, provided that a finite number of frequencies $k$ are chosen in a fixed range. These conditions are independent of the coefficients, in contrast to the illuminations classically constructed by means of complex geometric optics solutions. This theory finds applications in several hybrid problems, where unknown parameters have to be imaged from internal power density measurements. As an example, we discuss the microwave imaging by ultrasound deformation technique, for which we prove new reconstruction formulae.
\end{abstract}

\section{INTRODUCTION}

Let $d=2$ or $d=3$ be the dimension of the ambient space, $\Omega \subseteq \mathbb{R}^{d}$ be a smooth bounded domain and consider the Helmholtz equation

$$
\left\{\begin{array}{l}
-\operatorname{div}\left(a \nabla u_{k}^{\varphi}\right)-k q u_{k}^{\varphi}=0 \quad \text { in } \Omega \\
u_{k}^{\varphi}=\varphi \quad \text { on } \partial \Omega,
\end{array}\right.
$$

where $a$ is a real and symmetric tensor satisfying the ellipticity condition and $q>0$. Let $K_{\min }<$ $K_{\max }$ be the bounds for the possible values of $k$ and set $K_{a d}=\left[K_{\min }, K_{\max }\right]$. Problem (10) is well-posed provided that $k \notin \Sigma$, the set of the Dirichlet eigenvalues.

We want to find suitable illuminations $\varphi_{i}$ such that the corresponding solutions to (11) and their gradients satisfy certain non-zero properties inside the domain, e.g.

$$
\left|u_{k}^{\varphi_{1}}(x)\right|>0, \quad\left|\nabla u_{k}^{\varphi_{2}}(x)\right|>0
$$

and certain linear independence properties, e.g.

$$
\operatorname{det}\left[\begin{array}{lll}
\nabla u_{k}^{\varphi_{2}} & \cdots & \nabla u_{k}^{\varphi_{d+1}}
\end{array}\right](x)>0, \quad \operatorname{det}\left[\begin{array}{ccc}
u_{k}^{\varphi_{1}} & \ldots & u_{k}^{\varphi_{d+1}} \\
\nabla u_{k}^{\varphi_{1}} & \cdots & \nabla u_{k}^{\varphi_{d+1}}
\end{array}\right](x)>0 .
$$

As discussed more precisely below, these conditions are motivated by the reconstruction algorithms of several hybrid imaging techniques. The problem is usually set for a fixed frequency $k \in K_{a d} \backslash \Sigma$.

The classical way to tackle this problem is by means of the so called complex geometric optics solutions [30, 15]. These are particular high oscillatory solutions of the Helmholtz equation and can be used to determine suitable illuminations [14, 13. However, they can only be constructed when the parameters are sufficiently smooth. Furthermore, and most importantly, their construction

Date: September 24, 2013.

2000 Mathematics Subject Classification. 35R30, 35J57, 35B30, 35B38.

Key words and phrases. hybrid imaging, sets of measurements, power density measurements, critical points, multiple frequency, microwave imaging, ultrasound deformation, exact reconstruction formulae. 
depends on the coefficients $a$ and $q$. Thus, this cannot be considered a completely satisfactory answer to this issue: in inverse problems these are usually unknown. In the case $k=0$, the absence of critical points is studied in [2, 3].

The main contribution of this work is a very different approach to this problem by means of a multi-frequency approach. We shall give a priori, i.e. independent of $a$ and $q$, conditions on the illuminations whose corresponding solutions satisfy the required properties, provided a finite number of frequencies are used in the range $K_{a d}$. More precisely, there exist illuminations $\varphi_{i}$ and a finite number of frequencies $K \subseteq K_{a d} \backslash \Sigma$ such that (2) and (3) are satisfied for every $x \in \Omega$ and for some $k \in K$ depending on $x$. For example, we shall show that if $\Omega$ is convex then the choice $\left\{1, x_{1}, x_{2}\right\}$ is sufficient in two dimensions, and the same is true for $\left\{1, x_{1}, x_{2}, x_{3}\right\}$ in three dimensions, provided that $a$ is close to a constant. The main idea behind this method is simple: if the illuminations are suitably chosen then the zero level sets of functionals depending on $u_{k}^{\varphi}$ and $\nabla u_{k}^{\varphi}$ move when the frequency changes. The proof is based on the analyticity of the map $k \mapsto u_{k}^{\varphi}$. Note that an infinite number of frequencies and analyticity properties have been used in similar contexts [1, 18], but here only a finite number of $k$ is needed. Simplified versions of the main results can be found in Subsection 2.1.

The theory presented in this paper finds its applications in several hybrid imaging techniques. By combining measurements coming from two different modalities it is possible to obtain highresolution and high-contrast images. For a review of the state of the art in hybrid techniques, the reader is referred to the recent works [4, 12, 34, 26, 13. Generally, a hybrid problem involves two steps. First, internal energies are measured inside the domain and, second, from their knowledge the unknown parameters have to be reconstructed.

Many hybrid problems are governed by the Helmholtz equation (11) or by one of its variants (complex coefficients, different type of boundary conditions, for which a multi-frequency approach can be carried out in the same way), e.g. microwave imaging by ultrasound deformation [31, 17, quantitative thermo-acoustic [15, 10, transient elastography and magnetic resonance elastography [8, 27, 17] (for which the Helmholtz equation is used as a one-dimensional approximation). The internal energies are always linear or quadratic functionals of $u_{k}^{\varphi}$ and of $\nabla u_{k}^{\varphi}$ and the parameters $a$ and $q$ have to be imaged. From the reconstruction procedures discussed in these papers, it turns out that some or all the conditions (2) and (3) are necessary. Thus, being able to determine suitable illuminations independently of the unknown parameters is fundamental, and these can be given by the multi-frequency approach developed here. It is worth observing that the nature of this approach, for which the frequency $k$ depends on the points in the domain, is better suited in cases where the internal data are collected locally, as for instance in microwave imaging by ultrasound deformation. On the other hand, it is more restrictive when the internal data are measured in the whole domain, as in thermo-acoustic imaging. Namely, we will have redundant measurements in some parts of the domain, where (2) and (3) are satisfied for two or more frequencies.

As an example, we apply the theory to one of these hybrid problems, microwave imaging by ultrasound deformation, which was introduced in [7]. The internal energies have the form

$$
E=a\left|\nabla u_{k}^{\varphi}\right|^{2}, \quad e=q\left(u_{k}^{\varphi}\right)^{2},
$$

and the electromagnetic parameters $a$ and $q$ have to be reconstructed. We provide reconstruction formulae for $a / q$ and $q$, which are applicable if (2) and a weaker version of (3) are satisfied for a suitable set of illuminations and a finite number of frequencies in the microwave regime.

We believe that the multi-frequency approach developed in this work can be used in other contexts where conditions similar to (2) and (3) naturally arise [17. In many situations one looks 
for solutions of the Helmholtz equation satisfying certain properties: complex geometric optics is the only tool that has been used so far 13 and presents the difficulties described above. The proof of the results uses regularity results for the solutions, the analyticity of $k \mapsto u_{k}^{\varphi}$, the possibility of using several frequencies in a fixed range and the fact that for a certain value of the frequency (e.g. for $k=0$ ) the required conditions are satisfied. Therefore, as long as these requirements can be fulfilled, the multi-frequency approach can work also with other governing equations. The Helmholtz equation is not the only context where this theory may be applicable.

This paper is organized as follows. In Section 2 we precisely describe the setting and state the main theoretical results. Then, we apply these to a particular hybrid problem and provide reconstruction formulae. In Section 3 we consider the mathematical aspects of the Helmholtz equation with complex $k$ : we review well-posedness and regularity results and show the analyticity of the map $k \mapsto u_{k}^{\varphi}$. In Section 4 the multi-frequency approach is discussed and used to prove the main theorems. Section 5 is devoted to the study of the example and to the proof of the reconstruction formulae.

Let us clarify some notation. The space of infinitely differentiable functions compactly supported in $\Omega$ will be denoted by $\mathcal{D}(\Omega)$. We use the classical notation for function spaces and, unless otherwise stated, we always consider real-valued function spaces. Whenever complex-valued functions have to be taken, we add the letter $\mathbb{C}$, as for instance in $H^{1}(\Omega ; \mathbb{C})$. Let $\gamma$ denote the trace operator from $H^{1}(\Omega ; \mathbb{C})$ to $H^{1 / 2}(\partial \Omega ; \mathbb{C})$. In a Hilbert space $H$, the scalar product will be denoted by $(,)_{H}$. We shall use the notation $\theta_{u, v}$ for the angle between two non-zero vectors $u, v$ in a Hilbert space, i.e. $\theta_{u, v}=\arccos \frac{(u, v)}{\|u\|\|v\|}$. We write $B(x, r)$ for the ball centered in $x \in \mathbb{R}^{d}$ of radius $r>0$.

\section{MAin Results}

Let $d=2$ or 3 be the dimension of the ambient space, $\Omega^{\prime} \subseteq \Omega \subseteq \mathbb{R}^{d}$ be two $\mathcal{C}^{1, \alpha}$ bounded domains for some $\alpha \in(0,1)$. Let $a$ be a real, symmetric tensor satisfying the ellipticity condition

$$
\lambda|\xi|^{2} \leq \xi \cdot a \xi \leq \Lambda|\xi|^{2}, \quad \xi \in \mathbb{R}^{d},
$$

for some $\lambda, \Lambda>0$. We also suppose that $a$ is of class $\mathcal{C}^{0, \alpha}\left(\bar{\Omega} ; \mathbb{R}^{d \times d}\right)$. Let $q \in L^{\infty}(\Omega)$ satisfy

$$
\beta_{1} \leq q \leq \beta_{2} \text { almost everywhere in } \Omega,
$$

for some $\beta_{1}, \beta_{2}>0$. Let $\Sigma$ denote the set of the Dirichlet eigenvalues of the problem

$$
\left\{\begin{array}{l}
-\operatorname{div}\left(a \nabla u_{k}^{\varphi}\right)-k q u_{k}^{\varphi}=0 \quad \text { in } \Omega \\
u_{k}^{\varphi}=\varphi \quad \text { on } \partial \Omega,
\end{array}\right.
$$

which is a countable set of positive numbers going to infinity. For any $k \in \mathbb{C} \backslash \Sigma$ problem (1) is well-posed and $u_{k}^{\varphi} \in \mathcal{C}^{1}\left(\bar{\Omega} ; \mathbb{C}\right.$ ) (see Section 3 ). Let $K_{\min }<K_{\max }$ be the bounds for the possible wavenumbers $k$ and denote $K_{a d}=\left[K_{\min }, K_{\max }\right]$. More generally, we can assume that $K_{a d}$ is a nonempty continuous curve in $\mathbb{C}$, containing the admissible values for the frequencies $k$ and depending on the particular problem under consideration.

In this section we summarize the main results of this paper. In Subsection 2.1 the theoretical results regarding non-zero properties of solutions of the Helmholtz are discussed. In Subsection 2.2 we apply the general theory to a particular case.

2.1. A Multiple Frequency Approach to the Boundary Control of the Helmholtz Equation. Given finite subsets $K \subseteq K_{a d} \backslash \Sigma$ and $I \subseteq \gamma\left(\mathcal{C}^{1, \alpha}(\bar{\Omega})\right)$, the Cartesian product $K \times I$ is called a set of measurements. 
Given a set of measurements $K \times\left\{\varphi_{i}\right\}$, we consider the unique solution $u_{k}^{i} \in \mathcal{C}^{1}(\bar{\Omega})$ to the problem

$$
\left\{\begin{array}{l}
-\operatorname{div}\left(a \nabla u_{k}^{i}\right)-k q u_{k}^{i}=0 \\
u_{k}^{i}=\varphi_{i} \quad \text { on } \partial \Omega .
\end{array} \quad \text { in } \Omega,\right.
$$

The introduction of the following class of sets of measurements is motivated by several hybrid techniques [31, 15, 7, 10]. In this work we will not apply the full generality of this concept (see Definition 2.5].

Definition 2.1. Let $p, r, s>0$. A set of measurements $K \times\left\{\varphi_{i}: i=1, \ldots, d+1\right\}$ is complete in $\Omega^{\prime}$ if for every $x \in \Omega^{\prime}$ there exists $\bar{k}=\bar{k}(x) \in K$ such that:

$$
\begin{gathered}
\left|u_{\bar{k}}^{1}(x)\right| \geq p, \\
\left|\operatorname{det}\left[\begin{array}{ccc}
\nabla u_{\bar{k}}^{2} & \cdots & \nabla u_{\bar{k}}^{d+1}
\end{array}\right](x)\right| \geq r, \\
\left|\operatorname{det}\left[\begin{array}{ccc}
u_{\bar{k}}^{1} & \cdots & u_{\bar{k}}^{d+1} \\
\nabla u_{\bar{k}}^{1} & \cdots & \nabla u_{\bar{k}}^{d+1}
\end{array}\right](x)\right| \geq s .
\end{gathered}
$$

The conditions (CSM 1) and CSM 2 guarantee that meaningful power density measurements exist in every point. Further, (CSM 2 and CSM 3 imply that there are $d$ and $d+1$ independent measurements everywhere, respectively.

The existence of complete sets of measurements is a non trivial problem for general $a$ and $q$. In the references cited above, some or all these conditions are shown by using complex geometric optics solutions [30. In view of Proposition 3.3 in [16], suitable illuminations can be constructed with a fixed frequency $k_{0}$, provided that $a$ and $q$ are smooth enough. However, their construction depends on the knowledge of $a$ and $q$, that in the inverse problems we have in mind are unknown.

In order to tackle this issue, in Section 4 we discuss a multiple frequency approach to construct complete sets of measurements. The conditions on the illuminations are independent of the coefficients. Here we provide a simplified version of the main results therein discussed.

The next theorem discusses the construction of complete sets of measurements in dimension $d=2$. It is a particular case of Theorem 4.8

Theorem 2.2. Suppose $d=2, a \in \mathcal{C}^{0,1}(\bar{\Omega})$ and that $\Omega$ is convex and $\mathcal{C}^{2}$. Then we can choose a finite $K \subseteq K_{a d} \backslash \Sigma$ such that

$$
K \times\left\{1, x_{1}, x_{2}\right\}
$$

is a complete set of measurements in $\Omega$.

The next theorem deals with the construction of complete sets of measurements in dimension $d=3$. It is a particular case of Theorem 4.12 .

Theorem 2.3. Suppose $d=3$. Let $a_{0}$ be a constant, symmetric and positive definite matrix. There exists $\delta>0$ such that for any $s \in \mathcal{C}^{0, \alpha}\left(\bar{\Omega} ; \mathbb{R}^{d \times d}\right)$ with $\|s\|_{\mathcal{C}^{0, \alpha}\left(\bar{\Omega} ; \mathbb{R}^{d \times d}\right)} \leq \delta$ we can choose a finite $K \subseteq K_{a d} \backslash \Sigma$ such that

$$
K \times\left\{1, x_{1}, x_{2}, x_{3}\right\}
$$

is a complete set of measurements in $\Omega$ for $a=a_{0}+s$.

The proofs of these statements can be found in Section 4, which also contains more general versions of the previous results. In particular, we shall see that in two dimensions the illuminations $1, x_{1}$ and $x_{2}$ represent a simple selection from a much wider class and the domain does not have to be convex. Moreover, we describe how to choose the finite set of frequencies $K$. 
2.2. Applications to Microwave Imaging by Ultrasound Deformation. We now discuss an example to justify the introduction of complete sets. In fact, for our purposes, a wider class of sets of measurements is sufficient.

We consider the hybrid problem arising from the combination of microwaves and ultrasounds which was introduced in [7]. Full details and the proofs of the results are given in Section [5] In addition to the previous assumptions, we suppose that $a$ is scalar-valued. In microwave imaging, $a$ is the inverse of the magnetic permeability, $q$ is the electric permittivity and $K_{a d}=\left[K_{\min }, K_{\max }\right]$, with $K_{\min }>0$, are the admissible frequencies in the microwave regime.

Given a set of measurements $K \times\left\{\varphi_{i}\right\}$ we consider power density measurements of the form

$$
e_{k}^{i j}=q u_{k}^{i} u_{k}^{j}, \quad E_{k}^{i j}=a \nabla u_{k}^{i} \cdot \nabla u_{k}^{j},
$$

where $u_{k}^{i}$ is given by (6). For simplicity, we denote $e_{k}=\left(e_{k}^{i j}\right)_{i j}$ and similarly for $E$. These internal energies have to be considered as known functions in $\Omega^{\prime}$.

Problem 2.4. Choose a suitable set of measurements $K \times\left\{\varphi_{i}\right\}$ and find $a$ and $q$ in $\Omega^{\prime}$ from the knowledge of $e_{k}^{i j}$ and $E_{k}^{i j}$ in $\Omega^{\prime}$.

Problem 2.4 is solved via two reconstruction formulae for $a / q$ and $q$, respectively, which we shall now describe. Their applicability is guaranteed if $K \times\left\{\varphi_{i}\right\}$ is a proper set of measurements in $\Omega^{\prime}$.

Definition 2.5. Let $p$ and $r$ be two positive constants. A set of measurements $K \times\left\{\varphi_{i}: i=1,2,3\right\}$ is proper in $\Omega^{\prime}$ if for every $x \in \Omega^{\prime}$ there exists $\bar{k}=\bar{k}(x) \in K$ such that:

$$
\begin{gathered}
\left|u_{\bar{k}}^{1}(x)\right| \geq p, \\
\left|\nabla u_{\bar{k}}^{2}(x)\right|\left|\nabla u_{\bar{k}}^{3}(x)\right|\left|\sin \theta_{\nabla u_{\bar{k}}^{2}, \nabla u_{\bar{k}}^{3}}(x)\right| \geq r .
\end{gathered}
$$

The collection of all proper sets of measurements in $\Omega^{\prime}$ with constants $p$ and $r$ will be denoted by $\mathcal{P}\left(\Omega^{\prime} ; p, r\right)$.

Clearly, the conditions characterizing a proper set are weaker than the conditions of a complete set: the main difference relies in the requirement of only two independent measurements in any dimension. Thus, the construction of proper sets can be easily achieved by using the Theorems of the previous subsection (in dimension three the illumination $x_{3}$ is not needed).

The next statement gives an exact formula for $a / q$. The formula was first derived in [7] for the two-dimensional case, and we have extended it to any dimension. We use the following notation for a matrix-valued function $M$ of size $N$

$$
\operatorname{tr}(M)=\sum_{i=1}^{N} M_{i i}, \quad|\nabla M|_{2}^{2}=\sum_{i, j=1}^{N}\left|\nabla M_{i j}\right|^{2} .
$$

Theorem 2.6. Take $p, r>0$ and $K \times\left\{\varphi_{i}\right\} \in \mathcal{P}\left(\Omega^{\prime} ; p, r\right)$. Suppose that

$$
\left|E_{k}^{i i}(x)\right|,\left|e_{k}^{i i}(x)\right| \leq b, \quad x \in \Omega^{\prime},
$$

for some $b>0$. Take $x \in \Omega^{\prime}$ and $\bar{k}$ as in Definition 2.5. Then there exists $C=C\left(\lambda, \beta_{1}, b\right)>0$ such that

$$
\frac{\operatorname{tr}\left(e_{\bar{k}}\right) \operatorname{tr}\left(E_{\bar{k}}\right)-\operatorname{tr}\left(e_{\bar{k}} E_{\bar{k}}\right)}{\operatorname{tr}\left(e_{\bar{k}}\right)^{2}}(x) \geq C p^{2} r^{4}
$$


and $a / q$ is given in terms of the data by

$$
\left|\nabla\left(e_{\bar{k}} / \operatorname{tr}\left(e_{\bar{k}}\right)\right)\right|_{2}^{2} \frac{a}{q}=2 \frac{\operatorname{tr}\left(e_{\bar{k}}\right) \operatorname{tr}\left(E_{\bar{k}}\right)-\operatorname{tr}\left(e_{\bar{k}} E_{\bar{k}}\right)}{\operatorname{tr}\left(e_{\bar{k}}\right)^{2}} \text { in } x .
$$

We now give an exact formula for $q$. If compared to the one described in [7, this one is valid in any dimension and does not require the set of measurements to be complete. It only requires that

$$
\operatorname{tr}(e):=\sum_{i, k} e_{k}^{i i} \geq c>0 \quad \text { in } \Omega^{\prime},
$$

which holds provided that the set of measurements satisfies (PSM 1).

Theorem 2.7. Let $K \times\left\{\varphi_{i}\right\}$ be a proper set of measurements. Suppose $q \in H^{1}(\Omega)$. Then $\log q$ is the unique solution to the problem

$$
\left\{\begin{array}{l}
-\operatorname{div}(G \operatorname{tr}(e) \nabla u)=-\operatorname{div}(G \nabla(\operatorname{tr}(e)))+2 \sum_{k, i}\left(E_{k}^{i i}-k e_{k}^{i i}\right) \quad \text { in } \Omega^{\prime} \\
u=\log q_{\mid \partial \Omega^{\prime}} \quad \text { on } \partial \Omega^{\prime} .
\end{array}\right.
$$

The previous result allows to reconstruct $q$ from the knowledge of $G=a / q$. Also, $q$ is supposed to be known on the boundary of $\Omega^{\prime}$, which is a reasonable assumption since we are mainly interested in detecting inclusions inside the domain. At this point, an obvious formula for $a$ is $a=G q$.

The two exact formulae provided by the previous Theorems use the derivatives of the data. As a consequence, in presence of noise, they may not give good quality images. Thus, an optimal control approach would be an efficient way to find better approximations of the coefficients, starting from the good guess given by the formulae described here. Details can be found in [7].

\section{The Helmholtz Equation}

In this section the Helmholtz equation (11) is discussed, where $a \in \mathcal{C}^{0, \alpha}\left(\bar{\Omega} ; \mathbb{R}^{d \times d}\right)$ and $q \in L^{\infty}(\Omega)$ are as in Section 2 and satisfy (4) and (5). First, we show that the problem is well-posed in the general situation of complex $k$. Then, regularity results are discussed. In order to do this, we use the classical results on elliptic problems. The aim of this analysis is the proof of the analyticity of the map $k \mapsto u_{k}^{\varphi}$, which is given in Subsection 3.2

3.1. Well-Posedness and Regularity. First of all, we study existence, uniqueness and stability with homogeneous Dirichlet boundary conditions. The space $H^{-1}(\Omega ; \mathbb{C})$ denotes the continuous antidual of $H_{0}^{1}(\Omega ; \mathbb{C})$.

Proposition 3.1. There exists $\Sigma=\left\{\lambda_{i}: i \in \mathbb{N}\right\} \subseteq \mathbb{R}_{+}$with $\lambda_{i} \rightarrow+\infty$ such that for $k \in \mathbb{C} \backslash \Sigma$ and $f \in H^{-1}(\Omega ; \mathbb{C})$ the equation

$$
-\operatorname{div}(a \nabla u)-k q u=f
$$

has a unique solution $u \in H_{0}^{1}(\Omega ; \mathbb{C})$ satisfying $\|u\|_{H_{0}^{1}} \leq C\|f\|_{H^{-1}}$ for some $C>0$ independent of $f$. Moreover, for fixed $f \in H^{-1}(\Omega ; \mathbb{C})$, the map

$$
k \in \mathbb{C} \backslash \Sigma \longmapsto u \in H_{0}^{1}(\Omega ; \mathbb{C})
$$

is analytic.

Proof. We give a sketch of the proof. Consider $L=-\operatorname{div}(a \nabla \cdot): H_{0}^{1}(\Omega ; \mathbb{C}) \rightarrow H^{-1}(\Omega ; \mathbb{C})$ and $M_{q}: L^{2}(\Omega ; \mathbb{C}) \rightarrow L^{2}(\Omega ; \mathbb{C})$ defined by $f \mapsto q f$. By Lax-Milgram Theorem and (4) the operator $L$ is invertible and we can consider $S:=L^{-1} M_{q}: H_{0}^{1}(\Omega ; \mathbb{C}) \rightarrow H_{0}^{1}(\Omega ; \mathbb{C})$, which by Kondrachov Compactness Theorem and (5) is compact, self-adjoint and positive. Hence $S$ has a countable set of 
eigenvalues $\left\{\eta_{i}>0: i \in \mathbb{N}\right\}$, with $\eta_{i} \rightarrow 0$. Define $\Sigma=\left\{\lambda_{i}=1 / \eta_{i}: i \in \mathbb{N}\right\}$ and take $k \in \mathbb{C} \backslash \Sigma$. Since (11) is equivalent to $(I-k S) u=L^{-1} f$, the first part follows. Finally, the analyticity of $k \mapsto u$ is a consequence of the so called Analytic Fredholm Theorem (see [29, Theorem 8.92]).

As a consequence, we immediately get the following result regarding the Dirichlet boundary value problem for the Helmholtz equation.

Corollary 3.2. Take $k \in \mathbb{C} \backslash \Sigma$. Then there exists $C=C(\Omega, a, q, k)>0$ such that for every $f \in H^{-1}(\Omega ; \mathbb{C})$ and $\varphi \in H^{1 / 2}(\partial \Omega ; \mathbb{C})$ the problem

$$
\left\{\begin{array}{l}
-\operatorname{div}(a \nabla u)-k q u=f \quad \text { in } \Omega, \\
u=\varphi \quad \text { on } \partial \Omega,
\end{array}\right.
$$

has a unique solution $u \in H^{1}(\Omega ; \mathbb{C})$ satisfying

$$
\|u\|_{H^{1}(\Omega ; \mathbb{C})} \leq C\left(\|\varphi\|_{H^{1 / 2}(\partial \Omega ; \mathbb{C})}+\|f\|_{H^{-1}(\Omega ; \mathbb{C})}\right) .
$$

Moreover, for fixed $f \in H^{-1}(\Omega ; \mathbb{C})$ and $\varphi \in H^{1 / 2}(\partial \Omega ; \mathbb{C})$, the map

$$
k \in \mathbb{C} \backslash \Sigma \longmapsto u \in H^{1}(\Omega ; \mathbb{C})
$$

is analytic.

Standard elliptic regularity theory allows us to study the regularity of the solution $u \in H^{1}(\Omega ; \mathbb{C})$ to (12).

Proposition 3.3. Take $k \in \mathbb{C} \backslash \Sigma, f \in L^{\infty}(\Omega ; \mathbb{C}), v \in \mathcal{C}^{1, \alpha}(\bar{\Omega} ; \mathbb{C})$ and write $\varphi=\gamma(v)$. Let $u \in$ $H^{1}(\Omega ; \mathbb{C})$ be the unique solution to $(12)$. Then $u \in \mathcal{C}^{1}(\bar{\Omega} ; \mathbb{C})$ and there exists $C=C(\Omega, a, q, k)>0$ such that

$$
\|u\|_{\mathcal{C}^{1}(\bar{\Omega} ; \mathbb{C})} \leq C\left(\|v\|_{\mathcal{C}^{1, \alpha}(\bar{\Omega} ; \mathbb{C})}+\|f\|_{\infty}\right) .
$$

Proof. We decompose $u, f, \varphi$ and $k$ into real and imaginary parts by writing $u=u_{R}+i u_{I}$, $f=f_{R}+i f_{I}, v=v_{R}+i v_{I}$ and $k=k_{R}+i k_{I}$. By testing the first equation of (12) against any $w \in H_{0}^{1}(\Omega ; \mathbb{R})$ and by taking real and imaginary parts we obtain

$$
\begin{cases}-\operatorname{div}\left(a \nabla u_{R}\right)-k_{R} q u_{R}=-k_{I} q u_{I}+f_{R}, & \gamma\left(u_{R}\right)=\varphi_{R}, \\ -\operatorname{div}\left(a \nabla u_{I}\right)-k_{R} q u_{I}=k_{I} q u_{R}+f_{I}, & \gamma\left(u_{I}\right)=\varphi_{I} .\end{cases}
$$

In the rest of the proof we will consider constants $c=c(\Omega, a, q, k)$. From usual elliptic regularity theory (e.g. (8.38) in [24]) there holds

$$
\left\|u_{R}\right\|_{\infty} \leq c\left(\|u\|_{2}+\|v\|_{\mathcal{C}^{1, \alpha}(\bar{\Omega} ; \mathbb{C})}+\|f\|_{\infty}\right)
$$

By arguing in the same way with $u_{I}$ we infer that

$$
\|u\|_{\infty} \leq c\left(\|u\|_{2}+\|v\|_{\mathcal{C}^{1, \alpha}(\bar{\Omega} ; \mathbb{C})}+\|f\|_{\infty}\right) .
$$

Therefore, by Corollary 8.35 in 24 we get that $u_{R}, u_{I} \in \mathcal{C}^{1}(\bar{\Omega})$ and that

$$
\left\|u_{R}\right\|_{\mathcal{C}^{1}(\bar{\Omega})} \leq c\left(\|u\|_{2}+\|v\|_{\mathcal{C}^{1, \alpha}(\bar{\Omega} ; \mathbb{C})}+\|f\|_{\infty}\right) .
$$

A similar inequality holds for $u_{I}$ and so

$$
\|u\|_{\mathcal{C}^{1}(\bar{\Omega} ; \mathbb{C})} \leq c\left(\|u\|_{2}+\|v\|_{\mathcal{C}^{1, \alpha}(\bar{\Omega} ; \mathbb{C})}+\|f\|_{\infty}\right) .
$$

Finally, in view of (13) we obtain (14). 
3.2. Analyticity Properties. First, we need the following lemma concerning the composition of analytic functions.

Lemma 3.4 (33]). Let $D \subseteq \mathbb{C}$ be open, $X, Y$ be Banach spaces, $f_{i}: D \rightarrow X$ be analytic maps for $i=1, \ldots, b$ and $g: X^{b} \rightarrow Y$ be multilinear and bounded. Then

$$
g \circ\left(f_{1}, \ldots, f_{b}\right): D \longrightarrow Y
$$

is analytic.

In this subsection we study the dependence of the solutions $u_{k}^{\varphi}$ on $k$. We come back to the original problem

$$
\left\{\begin{array}{l}
-\operatorname{div}\left(a \nabla u_{k}^{\varphi}\right)-k q u_{k}^{\varphi}=0 \quad \text { in } \Omega \\
u_{k}^{\varphi}=\varphi \quad \text { on } \partial \Omega,
\end{array}\right.
$$

for $k \in \mathbb{C} \backslash \Sigma$ and fixed $\varphi \in \gamma\left(\mathcal{C}^{1, \alpha}(\bar{\Omega})\right)$. By Corollary 3.2 there exists a unique solution $u_{k}^{\varphi} \in$ $H^{1}(\Omega ; \mathbb{C})$, that in view of Proposition 3.3 is in $\mathcal{C}^{1}(\bar{\Omega} ; \mathbb{C})$. We have already seen that $u_{k}^{\varphi}$ depends analytically on the wavenumber $k$ with respect to the norm of $H^{1}(\Omega ; \mathbb{C})$. We now want to show this with respect to the $\mathcal{C}^{1}(\bar{\Omega} ; \mathbb{C})$ norm. The proof follows the argument used by Calderón to prove that $u_{k}^{\varphi}$ depends analytically on $a$ if $k=0$ [25].

Proposition 3.5. Take $\varphi \in \gamma\left(\mathcal{C}^{1, \alpha}(\bar{\Omega})\right)$. Then the map

$$
T: \mathbb{C} \backslash \Sigma \longrightarrow \mathcal{C}^{1}(\bar{\Omega} ; \mathbb{C}), \quad k \longmapsto u_{k}^{\varphi}
$$

is analytic, where $u_{k}^{\varphi}$ is the unique solution to (1)).

Proof. For simplicity, we do not write the dependence on $\varphi$. Fix $k_{0} \in \mathbb{C} \backslash \Sigma$ : we shall prove that $T$ is analytic in $k_{0}$.

For $h \in \mathbb{C}$ define the operator $C_{h}: \mathcal{C}^{1}(\bar{\Omega} ; \mathbb{C}) \rightarrow L^{\infty}(\Omega ; \mathbb{C}), w \mapsto h q w$ with norm $\left\|C_{h}\right\| \leq \beta_{2}|h|$. In view of Proposition 3.3, there exists a bounded operator $B: L^{\infty}(\Omega ; \mathbb{C}) \longrightarrow \mathcal{C}^{1}(\bar{\Omega} ; \mathbb{C})$, where $B f$ is the unique solution to

$$
\left\{\begin{array}{l}
-\operatorname{div}(a \nabla u)-k q u=f \quad \text { in } \Omega, \\
u=0 \quad \text { on } \partial \Omega .
\end{array}\right.
$$

As a consequence, $B C_{h}: \mathcal{C}^{1}(\bar{\Omega} ; \mathbb{C}) \rightarrow \mathcal{C}^{1}(\bar{\Omega} ; \mathbb{C})$ is a bounded operator with norm $\left\|B C_{h}\right\| \leq \beta_{2}\|B\||h|$. Define $r=\min \left\{1 /\left(\beta_{2}\|B\|\right), d\left(k_{0}, \Sigma\right)\right\}$, where $d\left(k_{0}, \Sigma\right)=\inf \left\{d\left(k_{0}, \sigma\right): \sigma \in \Sigma\right\}$ denotes the distance between $k_{0}$ and $\Sigma$. Take $k \in B\left(k_{0}, r\right) \subseteq \mathbb{C} \backslash \Sigma$. The increment $h=k-k_{0}$ satisfies $|h|<r \leq \frac{1}{\beta_{2}\|B\|}$, whence $\left\|B C_{h}\right\|<1$. Denoting $v=u_{k}-u_{k_{0}}$, by definition of $T$ we have

$$
-\operatorname{div}(a \nabla v)-k_{0} q v-h q v=h q u_{k_{0}}=C_{h} u_{k_{0}}
$$

which, after applying $B$ to both sides, becomes $\left(I-B C_{h}\right) v=B C_{h} u_{k_{0}}$, where $I$ denotes the identity operator of $\mathcal{C}^{1}(\bar{\Omega} ; \mathbb{C})$. As a result, since $\left\|B C_{h}\right\|<1$, we obtain

$$
v=\left(I-B C_{h}\right)^{-1} B C_{h} u_{k_{0}}=\sum_{n=1}^{\infty}\left(B C_{h}\right)^{n} u_{k_{0}} .
$$

It follows that

$$
T(k)=\sum_{n=0}^{\infty}\left(B C_{k-k_{0}}\right)^{n} T\left(k_{0}\right), \quad k \in B\left(k_{0}, r\right) .
$$

Namely, the map $T$ is analytic. 
For the sake of completeness, we now show that the dependence on $k$ of the internal energies (77) is analytic. Consider two different boundary data $\varphi_{1}, \varphi_{2} \in H^{1 / 2}(\partial \Omega ; \mathbb{C})$ and the corresponding solutions $u_{k}^{i}:=u_{k}^{\varphi_{i}}$ to (11). For $i, j=1,2$ we define the internal energies as

$$
\begin{array}{rlrl}
e^{i j}: & \mathbb{C} \backslash \Sigma \longrightarrow L^{1}(\Omega ; \mathbb{C}), & E^{i j}: \mathbb{C} \backslash \Sigma \longrightarrow L^{1}(\Omega ; \mathbb{C}), \\
& k \longmapsto q u_{k}^{i} u_{k}^{j}, & k & \longmapsto a \nabla u_{k}^{i} \cdot \nabla u_{k}^{j} .
\end{array}
$$

Theorem 3.6. Take $\varphi_{1}, \varphi_{2} \in H^{1 / 2}(\partial \Omega ; \mathbb{C})$. Then the internal energies $e^{i j}$ and $E^{i j}$ are analytic functions.

Proof. Note that the maps

$$
\begin{aligned}
g_{1}: H^{1}(\Omega ; \mathbb{C})^{2} & \longrightarrow L^{1}(\Omega ; \mathbb{C}), & g_{2}: H^{1}(\Omega ; \mathbb{C})^{2} & \longrightarrow L^{1}(\Omega ; \mathbb{C}) \\
(u, v) & \longmapsto q u v & (u, v) & \longmapsto a \nabla u \cdot \nabla u
\end{aligned}
$$

are bilinear and bounded. Therefore the result follows by Corollary 3.2 and Lemma 3.4 .

As a consequence of the unique continuation property for holomorphic functions we obtain the following

Corollary 3.7. Take $\varphi_{1}, \varphi_{2} \in H^{1 / 2}(\partial \Omega ; \mathbb{C})$. Let $k_{l}, k \in \mathbb{C} \backslash \Sigma$ such that $k_{l} \rightarrow k$ and $k_{l} \neq k$ for every $l \in \mathbb{N}$. If $e^{i j}\left[\right.$ resp. $\left.E^{i j}\right]$ is known in $k_{l}$ for every $l \in \mathbb{N}$ then $e^{i j}\left[\right.$ resp. $\left.E^{i j}\right]$ is known everywhere in $\mathbb{C} \backslash \Sigma$.

Remark 3.8. This result must be seen in view of Problem 2.4. The knowledge of $E^{i j}$ for infinitely many frequency in a fixed range determines $E^{i j}$ for $k=0$, where the reconstruction process for $a$ has been studied thoroughly [5, 9, 14, 22, 28, 34. However, this has to be regarded simply as an interesting theoretical result: analytic continuation is a very ill-posed process.

\section{A Multiple Frequency Approach to the Boundary Control of the Helmholtz EQUATION}

In this section we discuss the multi-frequency approach to the issue of the existence of complete and proper sets of measurements. The theorems stated in Subsection 2.1 will be a consequence of the results presented here.

4.1. Preliminaries. Let $a \in \mathcal{C}^{0, \alpha}\left(\bar{\Omega} ; \mathbb{R}^{d \times d}\right)$ and $q \in L^{\infty}(\Omega)$ be as in Section 2 and satisfy (4) and (5). Recall that $K_{a d}$ is the set for the possible wavenumber, namely we are allowed to choose values $k \in K_{a d} \backslash \Sigma$. As in Proposition 3.5, we denote the unique solution to the boundary value problem (11) by $u_{k}^{\varphi}=T^{\varphi}(k) \in \mathcal{C}^{1}(\bar{\Omega} ; \mathbb{C})$

(1)

$$
\left\{\begin{array}{l}
-\operatorname{div}\left(a \nabla u_{k}^{\varphi}\right)-k q u_{k}^{\varphi}=0 \quad \text { in } \Omega \\
u_{k}^{\varphi}=\varphi \quad \text { on } \partial \Omega
\end{array}\right.
$$

As a consequence of the analyticity of $k \mapsto u_{k}^{\varphi}$ proved in Proposition 3.5 we obtain the following

Lemma 4.1. Take $\Omega^{\prime} \subseteq \Omega, b \in \mathbb{N}^{*}$ and $\zeta: \mathcal{C}^{1}(\bar{\Omega} ; \mathbb{C})^{b} \rightarrow \mathcal{C}\left(\overline{\Omega^{\prime}} ; \mathbb{C}\right)$ multilinear and bounded. Let $\varphi_{1}, \ldots, \varphi_{b} \in \gamma\left(\mathcal{C}^{1, \alpha}(\bar{\Omega})\right)$ be such that

$$
\zeta\left(u_{k^{x}}^{\varphi_{1}}, \ldots, u_{k^{x}}^{\varphi_{b}}\right)(x) \neq 0, \quad x \in \overline{\Omega^{\prime}}
$$


for some $k^{x} \in \mathbb{C} \backslash \Sigma$. Take $k_{l}, k \in \mathbb{C} \backslash \Sigma$ with $k_{l} \rightarrow k$ and $k_{l} \neq k$. Then there exists a finite $L \subseteq \mathbb{N}$ such that

$$
\sum_{l \in L}\left|\zeta\left(u_{k_{l}}^{\varphi_{1}}, \ldots, u_{k_{l}}^{\varphi_{b}}\right)(x)\right|>0, \quad x \in \overline{\Omega^{\prime}}
$$

In particular, we can choose a finite $K \subseteq K_{a d} \backslash \Sigma$ such that

$$
\sum_{k \in K}\left|\zeta\left(u_{k}^{\varphi_{1}}, \ldots, u_{k}^{\varphi_{b}}\right)(x)\right|>0, \quad x \in \overline{\Omega^{\prime}}
$$

Remark 4.2. For the sake of completeness, we note that the statement holds true for $\zeta$ analytic, but our current applications do not need this extension. We shall use this lemma only with $k^{x}=0$. However, this more general version allows other possible applications. In particular, the case $\left|k^{x}\right| \rightarrow$ $\infty$ could be studied. This could be considered in the context of high-frequency approximations of the Helmholtz equation.

Remark 4.3. If in addition to the assumptions of this lemma we suppose that the set $\left\{x \in \overline{\Omega^{\prime}}\right.$ : $\left.\zeta\left(u_{k}^{\varphi_{1}}, \ldots, u_{k}^{\varphi_{b}}\right)(x)=0\right\}$ is finite, then there exists $\bar{l} \in \mathbb{N}$ such that

$$
\left|\zeta\left(u_{k}^{\varphi_{1}}, \ldots, u_{k}^{\varphi_{b}}\right)(x)\right|+\left|\zeta\left(u_{k_{\bar{l}}}^{\varphi_{1}}, \ldots, u_{k_{\bar{l}}}^{\varphi_{b}}\right)(x)\right|>0, \quad x \in \overline{\Omega^{\prime}} .
$$

Namely, if the zeros of $\zeta\left(u_{k}^{\varphi_{1}}, \ldots, u_{k}^{\varphi_{b}}\right)$ are isolated points then only two frequencies are necessary to obtain a non-zero quantity everywhere. We leave the proof of this extension to the reader.

Proof. Take $x \in \overline{\Omega^{\prime}}$. The map $e v_{x}: \mathcal{C}\left(\overline{\Omega^{\prime}} ; \mathbb{C}\right) \rightarrow \mathbb{C}, u \mapsto u(x)$ is linear and bounded. Hence, using Proposition 3.5 and Lemma 3.4 the map

$$
z_{x}:=e v_{x} \circ \zeta \circ\left(T^{\varphi_{1}}, \ldots, T^{\varphi_{b}}\right): \mathbb{C} \backslash \Sigma \longrightarrow \mathbb{C}
$$

is analytic. By (16) we have $z_{x}\left(k^{x}\right) \neq 0$, and so the set $\left\{k^{\prime} \in \mathbb{C} \backslash \Sigma: z_{x}\left(k^{\prime}\right)=0\right\}$ has no accumulation points in $\mathbb{C} \backslash \Sigma$ by the analytic continuation theorem. Since by assumption $k$ is an accumulation point for the sequence $\left(k_{l}\right)$, this implies that there exists $l_{x} \in \mathbb{N}$ such that $z_{x}\left(k_{l_{x}}\right) \neq 0$. Since $\zeta \circ\left(T^{\varphi_{1}}, \ldots, T^{\varphi_{b}}\right)\left(k_{l_{x}}\right)$ is continuous, we can find $r_{x}>0$ such that

$$
z_{y}\left(k_{l_{x}}\right) \neq 0, \quad y \in B\left(x, r_{x}\right) \cap \overline{\Omega^{\prime}} .
$$

Since $\overline{\Omega^{\prime}}=\bigcup_{x \in \overline{\Omega^{\prime}}}\left(B\left(x, r_{x}\right) \cap \overline{\Omega^{\prime}}\right)$ there exist $x_{1}, \ldots, x_{N} \in \overline{\Omega^{\prime}}$ satisfying

$$
\overline{\Omega^{\prime}}=\bigcup_{i=1}^{N}\left(B\left(x_{i}, r_{x_{i}}\right) \cap \overline{\Omega^{\prime}}\right) .
$$

Defining $L=\left\{l_{x_{i}}: i=1:, \ldots, N\right\}$, by (19) and (20) we obtain (17).

Let us now recall the definition of complete sets of measurements.

Definition 2.1. Let $p, r, s>0$. A set of measurements $K \times\left\{\varphi_{i}: i=1, \ldots, d+1\right\}$ is complete in $\Omega^{\prime}$ if for every $x \in \Omega^{\prime}$ there exists $\bar{k}=\bar{k}(x) \in K$ such that:

$$
\begin{aligned}
& \left|u_{\bar{k}}^{1}(x)\right| \geq p, \\
& \left|\operatorname{det}\left[\begin{array}{lll}
\nabla u_{\bar{k}}^{2} & \cdots & \nabla u_{\bar{k}}^{d+1}
\end{array}\right](x)\right| \geq r, \\
& \left|\operatorname{det}\left[\begin{array}{ccc}
u_{\bar{k}}^{1} & \cdots & u_{\bar{k}}^{d+1} \\
\nabla u_{\bar{k}}^{1} & \cdots & \nabla u_{\bar{k}}^{d+1}
\end{array}\right](x)\right| \geq s .
\end{aligned}
$$


In order to satisfy these conditions, the main idea is to trace back to the case $k=0$, where things are simpler. For instance, consider condition (CSM 1). With $k=0$, by the Strong Maximum Principle this is trivially satisfied provided that the boundary condition is strictly positive or negative. It remains to show that this good property transfers to any range of frequencies, and this is achieved with Lemma 4.1. In summary, the strategy to study these conditions can be outlined in the following three steps:

(1) choose a suitable $\zeta$ as above such that the condition we want to prove is equivalent to (18);

(2) prove the assumption (16) for $k^{x}=0$, which is in general easier than (18) since $k=0$;

(3) apply Lemma 4.1 to deduce the result.

We will deal with the issue of the construction of complete sets of measurements in two different situations, depending on the dimension. As we shall see, condition (CSM 1) will be a consequence of the Maximum Principle, that holds in any dimension and for any $a$. However, the study of conditions (CSM 2 and (CSM 3) in the case $k=0$ depends on the dimension.

4.2. Complete Sets: Two-Dimensional Case. Throughout this subsection we assume $d=2$. First, we consider conditions (CSM 1), (CSM 2) and (CSM 3) for $k=0$. As far as (CSM 1) is concerned, the Maximum Principle will be the main tool, and no further investigation is required.

Let us now focus on (CSM 2) for $k=0$, which reads

$$
\operatorname{det}\left[\begin{array}{ll}
\nabla u_{0}^{2} & \nabla u_{0}^{3}
\end{array}\right] \geq r
$$

for some $r>0$. This problem has been studied by Bauman et al. 19 in the context of univalent mappings. We introduce the following class of boundary conditions.

Definition 4.4. Let $\psi=\left(\psi_{2}, \psi_{3}\right) \in \mathcal{C}^{1, \alpha}\left(\bar{\Omega} ; \mathbb{R}^{2}\right)$ be a $\mathcal{C}^{1}$ diffeomorphism of a neighborhood of $\bar{\Omega}$ into $\mathbb{R}^{2}$ such that $J \psi>0$ in $\Omega$. Denote the restriction of $\psi$ to the boundary $\partial \Omega$ by $\varphi=\left(\varphi_{2}, \varphi_{3}\right):=\psi_{\mid \partial \Omega}$. We say that $\varphi_{2}, \varphi_{3}$ are $B M N$ boundary conditions if $\varphi: \partial \Omega \rightarrow \mathbb{R}^{2}$ maps $\partial \Omega$ onto a convex closed curve. In this case we write $\left(\varphi_{2}, \varphi_{3}\right) \in B M N(\Omega)$.

Remark 4.5. Note that if $\Omega$ is a convex domain then $\varphi_{2}=x_{1}, \varphi_{3}=x_{2}$ are BMN boundary conditions.

The main result in 19 states that (CSM 2) is satisfied for $k=0$, provided that the boundary conditions are BMN.

Proposition 4.6 (Theorems 2.4-2.5, [19]). Suppose $d=2$. If $\left(\varphi_{2}, \varphi_{3}\right) \in B M N(\Omega)$ then

$$
\operatorname{det}\left[\begin{array}{ll}
\nabla u_{0}^{2} & \nabla u_{0}^{3}
\end{array}\right]>0 \quad \text { in } \Omega \text {. }
$$

If in addition $\Omega$ is a $\mathcal{C}^{2}$ domain and $a \in \mathcal{C}^{0,1}\left(\bar{\Omega} ; \mathbb{R}^{d \times d}\right)$ then

$$
\operatorname{det}\left[\begin{array}{ll}
\nabla u_{0}^{2} & \nabla u_{0}^{3}
\end{array}\right]>0 \quad \text { in } \bar{\Omega} \text {. }
$$

Let us now focus on (CSM 3) for $k=0$. As far as the author is aware, there is no result concerning the zeros of

$$
\operatorname{det}\left[\begin{array}{ccc}
u_{0}^{1} & u_{0}^{2} & u_{0}^{3} \\
\nabla u_{0}^{1} & \nabla u_{0}^{2} & \nabla u_{0}^{3}
\end{array}\right] .
$$

Therefore we need the following statement, whose proof can be found at the end of this subsection. 
Lemma 4.7. Suppose $d=2$. Take $\varphi_{1}, \varphi_{2}, \varphi_{3} \in \gamma\left(\mathcal{C}^{1, \alpha}(\bar{\Omega})\right)$ such that $\varphi_{1}$ has a fixed sign and $\left(\varphi_{2}, \varphi_{3}\right),\left(\frac{\varphi_{2}}{\varphi_{1}}, \frac{\varphi_{3}}{\varphi_{1}}\right) \in B M N(\Omega)$. Then

$$
\operatorname{det}\left[\begin{array}{ccc}
u_{0}^{1} & u_{0}^{2} & u_{0}^{3} \\
\nabla u_{0}^{1} & \nabla u_{0}^{2} & \nabla u_{0}^{3}
\end{array}\right] \neq 0 \quad \text { in } \Omega .
$$

If in addition $\Omega$ is a $\mathcal{C}^{2}$ domain and $a \in \mathcal{C}^{0,1}\left(\bar{\Omega} ; \mathbb{R}^{d \times d}\right)$ then

$$
\operatorname{det}\left[\begin{array}{ccc}
u_{0}^{1} & u_{0}^{2} & u_{0}^{3} \\
\nabla u_{0}^{1} & \nabla u_{0}^{2} & \nabla u_{0}^{3}
\end{array}\right] \neq 0 \quad \text { in } \bar{\Omega} .
$$

We are now in a position to state the main results of this subsection, concerning the construction of complete sets of measurements in dimension $d=2$.

Theorem 4.8. Suppose $d=2$. Take $\Omega^{\prime} \Subset \Omega$ and $\varphi_{1}, \varphi_{2}, \varphi_{3} \in \gamma\left(\mathcal{C}^{1, \alpha}(\bar{\Omega})\right)$ such that $\varphi_{1}$ has a fixed sign and $\left(\varphi_{2}, \varphi_{3}\right),\left(\frac{\varphi_{2}}{\varphi_{1}}, \frac{\varphi_{3}}{\varphi_{1}}\right) \in B M N(\Omega)$. We can choose a finite $K \subseteq K_{a d} \backslash \Sigma$ such that

$$
K \times\left\{\varphi_{1}, \varphi_{2}, \varphi_{3}\right\}
$$

is a complete set of measurements in $\Omega^{\prime}$.

If in addition $\Omega$ is a $\mathcal{C}^{2}$ domain and $a \in \mathcal{C}^{0,1}\left(\bar{\Omega} ; \mathbb{R}^{d \times d}\right)$ then we can choose a finite $K^{\prime} \subseteq K_{a d} \backslash \Sigma$ such that

$$
K^{\prime} \times\left\{\varphi_{1}, \varphi_{2}, \varphi_{3}\right\}
$$

is a complete set of measurements in $\Omega$.

Remark 4.9. Note that the hypotheses on the boundary conditions are satisfied if $\varphi_{1}=1$ and $\left(\varphi_{2}, \varphi_{3}\right) \in B M N(\Omega)$. Therefore, if $\Omega$ is convex, by Remark 4.5 an easy choice for the boundary conditions is $\varphi_{1}=1, \varphi_{2}=x_{1}$ and $\varphi_{3}=x_{2}$.

Proof. By Lemma 4.7, Proposition 4.6 and Strong Maximum Principle we obtain that

$$
u_{0}^{1}, \operatorname{det}\left[\begin{array}{cc}
\nabla u_{0}^{2} & \nabla u_{0}^{3}
\end{array}\right], \operatorname{det}\left[\begin{array}{ccc}
u_{0}^{1} & u_{0}^{2} & u_{0}^{3} \\
\nabla u_{0}^{1} & \nabla u_{0}^{2} & \nabla u_{0}^{3}
\end{array}\right] \neq 0 \quad \text { in } \Omega
$$

respectively. Thus we can apply Lemma 4.1 with $\Omega^{\prime} \Subset \Omega, b=6, k^{x}=0, \varphi_{4}=\varphi_{1}, \varphi_{5}=\varphi_{2}, \varphi_{6}=\varphi_{3}$

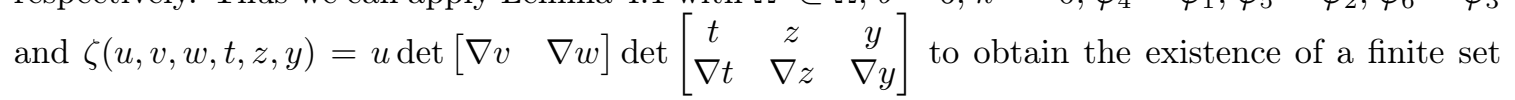
$K \subseteq K_{a d} \backslash \Sigma$ such that

$$
p(x):=\sum_{k \in K}\left|u_{k}^{1} \operatorname{det}\left[\begin{array}{ll}
\nabla u_{k}^{2} & \nabla u_{k}^{3}
\end{array}\right] \operatorname{det}\left[\begin{array}{ccc}
u_{k}^{1} & u_{k}^{2} & u_{k}^{3} \\
\nabla u_{k}^{1} & \nabla u_{k}^{2} & \nabla u_{k}^{3}
\end{array}\right]\right|(x)>0, \quad x \in \overline{\Omega^{\prime}} .
$$

In the sequel, we shall denote several positive constants independent of $x \in \overline{\Omega^{\prime}}$ by $c$. Since $u_{k}^{\varphi_{i}} \in$ $\mathcal{C}^{1}(\bar{\Omega})$ (Proposition [3.3), $p(x) \geq c$ for every $x \in \overline{\Omega^{\prime}}$. As a result, for any $x \in \Omega^{\prime}$ there exists $k \in K$ such that

As a consequence we have

$$
\left|u_{k}^{1} \operatorname{det}\left[\begin{array}{ll}
\nabla u_{k}^{2} & \nabla u_{k}^{3}
\end{array}\right] \operatorname{det}\left[\begin{array}{ccc}
u_{k}^{1} & u_{k}^{2} & u_{k}^{3} \\
\nabla u_{k}^{1} & \nabla u_{k}^{2} & \nabla u_{k}^{3}
\end{array}\right]\right|(x) \geq c .
$$

$$
\left|u_{k}^{1}(x)\right| \geq c, \quad\left|\operatorname{det}\left[\begin{array}{ll}
\nabla u_{k}^{2} & \nabla u_{k}^{3}
\end{array}\right](x)\right| \geq c, \quad\left|\operatorname{det}\left[\begin{array}{ccc}
u_{k}^{1} & u_{k}^{2} & u_{k}^{3} \\
\nabla u_{k}^{1} & \nabla u_{k}^{2} & \nabla u_{k}^{3}
\end{array}\right](x)\right| \geq c,
$$

whence the first part of the theorem follows. 
If in addition we suppose that $\Omega$ is a $\mathcal{C}^{2}$ domain and that $a \in \mathcal{C}^{0,1}\left(\bar{\Omega} ; \mathbb{R}^{d \times d}\right)$, we can use the second part of Proposition 4.6 and the second part of Lemma 4.7 to infer that

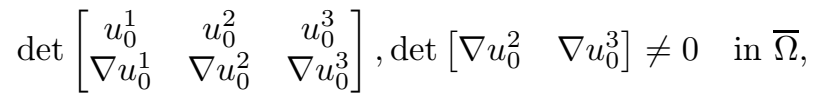

respectively. Therefore, arguing as before, we obtain a complete set in $\Omega$.

From the proof of Theorem 4.8 it is clear that the assumptions $\varphi_{1} \gtrless 0$ and $\left(\varphi_{2}, \varphi_{3}\right) \in B M N(\Omega)$ allow us to deduce (CSM 1) and (CSM 22) of Definition 2.1] respectively. One may wonder if the above Theorem still holds without one of these two hypotheses. The answer is no, as the following examples show. Therefore, if the boundary conditions $\varphi_{i}$ are not chosen properly, in general one cannot expect to obtain (CSM 1) and (CSM 2) by doing many measurements with different wavenumbers but with fixed illuminations. First, we provide a counterexample for condition (CSM 1).

Example 4.10. Suppose $d=2, \Omega=B(0,1)$ and $a=q=1$. We choose the boundary condition $\varphi_{1}\left(x_{1}, x_{2}\right)=x_{1}$, that clearly does not have a constant sign. In this case the Helmholtz equation (11) can be written in polar coordinates $(r, \theta)$ and reads

$$
u_{r r}+\frac{u_{r}}{r}+\frac{u_{\theta \theta}}{r^{2}}+k u=0
$$

It is straightforward to see that

$$
u_{k}^{1}(r, \theta)=\frac{J_{1}(\sqrt{k} r)}{J_{1}(\sqrt{k})} \cos \theta
$$

satisfies the above equation, where $J_{1}$ is the Bessel function of the first kind of order 1 and $k>0$ is not an eigenvalue of the problem. Thus, $\left\{u_{k}^{1}\right\}$ represents a family of solution to the Helmholtz equation with fixed boundary condition and varying wavenumber. However, condition (CSM 1) cannot hold since $u_{k}^{1}\left(0, x_{2}\right)=0$ for every $k$ and for every $x_{2} \in(-1,1)$.

Next, we study condition (CSM 22). Since it expresses the linear independence of the gradient of the solutions inside the domain, we shall see that it is not possible to require $\varphi_{1}, \varphi_{2}$ to be just linearly independent.

Example 4.11. We consider the situation of Example 4.10. Suppose $\Omega=B(0,1)$ and $a=q=1$. We choose the boundary conditions $\varphi_{2}\left(x_{1}, x_{2}\right)=x_{1}$ and $\varphi_{3}=1$. Clearly, $\left(\varphi_{2}, \varphi_{3}\right) \notin B M N(\Omega)$ since $\varphi_{3}$ is a constant, but $\varphi_{2}, \varphi_{3}$ are linearly independent. It is straightforward to see that the corresponding solutions to (11) are

$$
u_{k}^{2}(r, \theta)=\frac{J_{1}(\sqrt{k} r)}{J_{1}(\sqrt{k})} \cos \theta, \quad u_{k}^{3}(r, \theta)=\frac{J_{0}(\sqrt{k} r)}{J_{0}(\sqrt{k})},
$$

where $J_{n}$ is the Bessel function of the first kind of order $n$ and $k>0$ is not an eigenvalue of the problem.

Take a matrix-valued function $A: \Omega \rightarrow G L(2)$, where $G L(2)$ denotes the set of $2 \times 2$ invertible matrices. By viewing $A(x)$ as a change of coordinates in $T_{x} \Omega$, the tangent space in $x$ to $\Omega$, we get

$$
\operatorname{det}\left[A \nabla u_{k}^{2} A^{-1} \quad A \nabla u_{k}^{3} A^{-1}\right]=\operatorname{det}\left(A\left[\begin{array}{ll}
\nabla u_{k}^{2} & \nabla u_{k}^{3}
\end{array}\right] A^{-1}\right)=\operatorname{det}\left[\begin{array}{ll}
\nabla u_{k}^{2} & \nabla u_{k}^{3}
\end{array}\right] .
$$

Therefore, as far as $\operatorname{det}\left[\begin{array}{ll}\nabla u_{k}^{2} & \nabla u_{k}^{3}\end{array}\right]$ is concerned, we can express the gradient in any system of coordinates. 
In this case, writing $\nabla u_{k}^{i}$ with respect to $e_{\theta}$ and $e_{r}$ we have $\nabla u_{k}^{i}=\frac{1}{r} \frac{\partial u_{k}^{i}}{\partial \theta} e_{\theta}+\frac{\partial u_{k}^{i}}{\partial r} e_{r}$. Hence

$$
\operatorname{det}\left[\begin{array}{ll}
\nabla u_{k}^{2} & \nabla u_{k}^{3}
\end{array}\right]=\operatorname{det}\left[\begin{array}{cc}
\frac{1}{r} \frac{\partial u_{k}^{2}}{\partial \theta} & \frac{1}{r} \frac{\partial u_{k}^{3}}{\partial \theta} \\
\cdot & \cdot
\end{array}\right]=\operatorname{det}\left[\begin{array}{cc}
-\frac{1}{r} \frac{J_{1}(\sqrt{k} r)}{J_{1}(\sqrt{k})} \sin \theta & 0 \\
\cdot & \cdot
\end{array}\right] .
$$

Thus, we have $\operatorname{det}\left[\begin{array}{ll}\nabla u_{k}^{2} & \nabla u_{k}^{3}\end{array}\right]\left(x_{1}, 0\right)=0$ for every $k$ and for every $x_{1} \in(-1,1)$ and so (CSM 2 cannot be satisfied by using many measurements with these fixed illuminations and varying wavenumbers.

We end this subsection with the proof of Lemma 4.7

Proof. (Lemma 4.7). We shall prove only the first part of the lemma. The second part can be proved following the same argument.

For simplicity of notation we write $u_{i}:=u_{0}^{i}$ and suppose $\varphi_{1}>0$. By Proposition 4.6 we have $\operatorname{det}\left[\begin{array}{ll}\nabla u_{2} & \nabla u_{3}\end{array}\right]>0$ in $\Omega$, and so $\left\{\nabla u_{2}, \nabla u_{3}\right\}$ form a basis of $\mathbb{R}^{2}$ in every point of $\Omega$. Therefore there exist $\lambda, \mu: \Omega \rightarrow \mathbb{R}$ such that

$$
\nabla u_{1}=\lambda \nabla u_{2}+\mu \nabla u_{3} \quad \text { in } \Omega,
$$

whence

$$
\operatorname{det}\left[\begin{array}{ccc}
u_{1} & u_{2} & u_{3} \\
\nabla u_{1} & \nabla u_{2} & \nabla u_{3}
\end{array}\right]=\left(u_{1}-\lambda u_{2}-\mu u_{3}\right) \operatorname{det}\left[\begin{array}{ccc}
\nabla u_{2} & \nabla u_{3}
\end{array}\right] \quad \text { in } \Omega .
$$

By contradiction, suppose that there exists $x_{0} \in \Omega$ such that

$$
u_{1}\left(x_{0}\right)=\lambda\left(x_{0}\right) u_{2}\left(x_{0}\right)+\mu\left(x_{0}\right) u_{3}\left(x_{0}\right) .
$$

Denote $\lambda_{0}=\lambda\left(x_{0}\right), \mu_{0}=\mu\left(x_{0}\right)$ and $z=\lambda_{0} u_{2}+\mu_{0} u_{3}$. Since $\varphi_{1}>0$, by the Strong Maximum Principle we get $u_{1}>0$ in $\bar{\Omega}$. Let $h=z / u_{1}$ and $\psi_{i}=u_{i} / u_{1}$ for $i=2,3$. For every $v \in \mathcal{D}(\Omega)$ and $i=2,3$ a straightforward calculation shows that (see Lemma 5.2)

$$
\int_{\Omega} u_{1}^{2} a \nabla \psi_{i} \cdot \nabla v d x=0
$$

Therefore $\psi_{i}$ is the unique solution to the problem

$$
\left\{\begin{array}{l}
-\operatorname{div}\left(u_{1}^{2} a \nabla \psi_{i}\right)=0 \quad \text { in } \Omega \\
\psi_{i}=\varphi_{i} / \varphi_{1}
\end{array}\right.
$$

and by using Proposition 4.6 we infer that

$$
\operatorname{det}\left[\begin{array}{ll}
\nabla \psi_{2} & \nabla \psi_{3}
\end{array}\right]>0 \quad \text { in } \Omega,
$$

since $\left(\frac{\varphi_{2}}{\varphi_{1}}, \frac{\varphi_{3}}{\varphi_{1}}\right) \in B M N(\Omega)$. By (21) and (22) we have $\nabla u_{1}\left(x_{0}\right)=\nabla z\left(x_{0}\right)$ and $u_{1}\left(x_{0}\right)=z\left(x_{0}\right)>0$, whence $\nabla\left(\log u_{1}\right)\left(x_{0}\right)=\nabla(\log z)\left(x_{0}\right)$ and so $\nabla(\log h)\left(x_{0}\right)=0$. As a consequence, $0=\nabla h\left(x_{0}\right)=$ $\lambda_{0} \nabla \psi_{2}\left(x_{0}\right)+\mu_{0} \nabla \psi_{3}\left(x_{0}\right)$, which contradicts (23) since $\left(\lambda_{0}, \mu_{0}\right) \neq(0,0)$.

4.3. Complete Sets: Three-Dimensional Case. Throughout this subsection we assume $d=3$. First, we need to consider (CSM 2) and (CSM 3) for $k=0$. In contrast to the case $d=2$, we cannot use Proposition 4.6] since the equivalent statement is known not to hold in three dimensions [20].

Thus, we assume that the parameter $a$ is a small perturbation of a constant, symmetric and positive definite matrix $a_{0}$, i.e.

$$
a=a_{0}+s, \quad\|s\|_{\mathcal{C}^{0, \alpha}\left(\bar{\Omega} ; \mathbb{R}^{d \times d}\right)} \leq \delta,
$$

with $\delta$ small enough. The study of this approximation is common in the literature [31, 15, 6, 10]. Note that we do not make any assumptions on $q$. 
In order to understand why this approximation is useful, let us consider the constant case, i.e. $a=a_{0}$. Choose $\varphi_{1}=1, \varphi_{2}=x_{1}, \varphi_{3}=x_{2}$ and $\varphi_{4}=x_{3}$. Hence $1, x_{1}, x_{2}$ and $x_{3}$ are the solutions to the problems

$$
\left\{\begin{array}{l}
-\operatorname{div}\left(a_{0} \nabla u\right)=0 \\
u=\varphi_{i} \quad \text { on } \partial \Omega,
\end{array} \text { in } \Omega\right.
$$

for $i=1, \ldots, 4$, respectively. Therefore conditions (CSM 1), (CSM 2) and (CSM 3) are trivially satisfied in the case $k=0$. Thanks to the multi-frequency approach we can extend this property to any range of frequencies, and a continuity argument allows small variations around a constant value. These two steps are carried out in the following theorem, which concerns the construction of complete sets of measurements in dimension $d=3$.

Theorem 4.12. Suppose $d=3$. Let $a_{0}$ be a constant, symmetric and positive definite matrix. There exists $\delta>0$ such that for any $s \in \mathcal{C}^{0, \alpha}\left(\bar{\Omega} ; \mathbb{R}^{d \times d}\right)$ with $\|s\|_{\mathcal{C}^{0, \alpha}\left(\bar{\Omega} ; \mathbb{R}^{d \times d}\right)} \leq \delta$ we can choose a finite $K \subseteq K_{a d} \backslash \Sigma$ such that

$$
K \times\left\{1, x_{1}, x_{2}, x_{3}\right\}
$$

is a complete set of measurements in $\Omega$ for $a=a_{0}+s$.

Proof. Fix $0<\varepsilon<\lambda / 3$, where $\lambda$ is the smallest eigenvalue of $a_{0}$. In the following we will consider positive constants $c$ depending on $a_{0}, \Omega$ and $\varepsilon$. Take $0<\delta \leq \varepsilon$ and $s \in \mathcal{C}^{0, \alpha}\left(\bar{\Omega} ; \mathbb{R}^{d \times d}\right)$ with $\|s\|_{\mathcal{C}^{0, \alpha}\left(\bar{\Omega} ; \mathbb{R}^{d \times d}\right)} \leq \delta$. For $a_{s}=a_{0}+s$ consider $u_{0}^{i}(s)$ the solution to the problem

$$
\left\{\begin{array}{l}
-\operatorname{div}\left(a_{s} \nabla u_{0}^{i}(s)\right)=0 \\
u_{0}^{i}(s)=\varphi_{i} \quad \text { on } \partial \Omega,
\end{array} \quad \text { in } \Omega,\right.
$$

where $\varphi_{1}=1$ and $\varphi_{i}=x_{i-1}$ for $i=2,3,4$. Thus, $\left\|u_{0}^{i}(s)\right\|_{2} \leq c$ for some $c>0$. As a result, from usual elliptic regularity theory (e.g. (8.38) in [24]) we obtain $\left\|u_{0}^{i}(s)\right\|_{\infty} \leq c$. By Theorem 8.33 in [24] this implies that

$$
\left\|\nabla u_{0}^{i}(s)\right\|_{\mathcal{C}^{0, \alpha}\left(\bar{\Omega} ; \mathbb{R}^{d}\right)} \leq c .
$$

The same argument applied to $u_{0}^{i}(s)-u_{0}^{i}(0)$ as a solution to

$$
-\operatorname{div}\left(a_{0} \nabla\left(u_{0}^{i}(s)-u_{0}^{i}(0)\right)\right)=\operatorname{div}\left(s \nabla u_{0}^{i}(s)\right) .
$$

gives $\left\|u_{0}^{i}(s)-u_{0}^{i}(0)\right\|_{\infty} \leq c \delta$ for some $c>0$. Hence, by (24) and Theorem 8.33 in [24] applied to $u_{0}^{i}(s)-u_{0}^{i}(0)$ there holds

$$
\left\|\nabla u_{0}^{i}(s)-\nabla u_{0}^{i}(0)\right\|_{\infty} \leq c\left(\left\|u_{0}^{i}(s)-u_{0}^{i}(0)\right\|_{\infty}+\left\|s \nabla u_{0}^{i}(s)\right\|_{\mathcal{C}^{0, \alpha}\left(\bar{\Omega} ; \mathbb{R}^{d}\right)}\right) \leq c \delta,
$$

whence

$$
\left\|u_{0}^{i}(s)-u_{0}^{i}(0)\right\|_{\mathcal{C}^{1}(\bar{\Omega})} \leq c \delta .
$$

As a consequence, since for $s=0$ we have

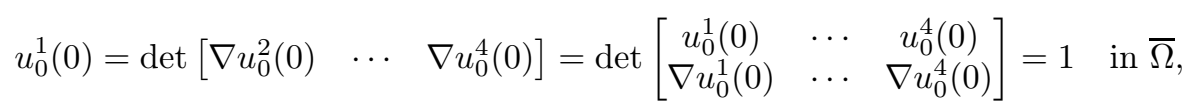

there exists $\delta \leq \varepsilon$ such that

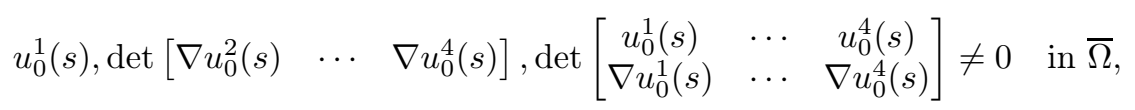

for all $s \in \mathcal{C}^{0, \alpha}\left(\bar{\Omega} ; \mathbb{R}^{d \times d}\right)$ with $\|s\|_{\mathcal{C}^{0, \alpha}\left(\bar{\Omega} ; \mathbb{R}^{d \times d}\right)} \leq \delta$. Finally, arguing as in the proof of Theorem 4.8, the result follows by using Lemma 4.1. 
4.4. Proper Sets of Measurements. This subsection is devoted to the study of proper sets of measurements, which were introduced in Subsection 2.2. Since the conditions characterizing proper sets are weaker than those of complete sets, we are not going into the details: the reader is referred to the previous parts of this section. We first recall the definition of proper sets of measurements for $a \in \mathcal{C}^{0, \alpha}\left(\bar{\Omega} ; \mathbb{R}^{d \times d}\right)$ and $q \in L^{\infty}(\Omega)$ satisfying (4) and (5).

Definition 2.5. Let $p$ and $r$ be two positive constants. A set of measurements $K \times\left\{\varphi_{i}: i=1,2,3\right\}$ is proper in $\Omega^{\prime}$ if for every $x \in \Omega^{\prime}$ there exists $\bar{k}=\bar{k}(x) \in K$ such that:

$$
\begin{gathered}
\left|u_{\bar{k}}^{\frac{1}{k}}(x)\right| \geq p, \\
\left|\nabla u_{\bar{k}}^{2}(x)\right|\left|\nabla u_{\bar{k}}^{3}(x)\right|\left|\sin \theta_{\nabla u_{\bar{k}}^{2}, \nabla u_{\bar{k}}^{\frac{3}{k}}}(x)\right| \geq r .
\end{gathered}
$$

The collection of all proper sets of measurements in $\Omega^{\prime}$ with constants $p$ and $r$ will be denoted by $\mathcal{P}\left(\Omega^{\prime} ; p, r\right)$.

The multi-frequency approach discussed in the last section is easily applicable for the construction of proper sets of measurements. In general, the choice for the illuminations $1, x_{1}$ and $x_{2}$ gives a proper set in any dimension (in dimension three, $a$ is required to be close to a constant).

Note that, in general, condition (PSM 2) is weaker than (CSM 2), and they are equivalent when $d=2$ since

$$
\left|\operatorname{det}\left(\left[\begin{array}{ll}
w & z
\end{array}\right]\right)\right|=|w||z|\left|\sin \theta_{w, z}\right|, \quad w, z \in \mathbb{R}^{2} .
$$

Thus, when $d=2$ one can directly use an analogue version of Theorem 4.8, without the assumption $\left(\frac{\varphi_{2}}{\varphi_{1}}, \frac{\varphi_{3}}{\varphi_{1}}\right) \in B M N(\Omega)$, which was needed to satisfy (CSM 3).

Theorem 4.13. Suppose $d=2$. Take $\Omega^{\prime} \Subset \Omega$ and $\varphi_{1}, \varphi_{2}, \varphi_{3} \in \gamma\left(\mathcal{C}^{1, \alpha}(\bar{\Omega})\right)$ such that $\varphi_{1}$ has a fixed sign and $\left(\varphi_{2}, \varphi_{3}\right) \in B M N(\Omega)$. We can choose a finite $K \subseteq K_{a d} \backslash \Sigma$ such that

$$
K \times\left\{\varphi_{1}, \varphi_{2}, \varphi_{3}\right\}
$$

is a proper set of measurements in $\Omega^{\prime}$.

If in addition $\Omega$ is a $\mathcal{C}^{2}$ domain and $a \in \mathcal{C}^{0,1}\left(\bar{\Omega} ; \mathbb{R}^{d \times d}\right)$ then we can choose a finite $K^{\prime} \subseteq K_{a d} \backslash \Sigma$ such that

$$
K^{\prime} \times\left\{\varphi_{1}, \varphi_{2}, \varphi_{3}\right\}
$$

is a proper set of measurements in $\Omega$.

In dimension three, it is straightforward to check that the analogue version of Theorem 4.12 is applicable, without the illumination $x_{3}$.

Theorem 4.14. Suppose $d=3$. Let $a_{0}$ be a constant, symmetric and positive definite matrix. There exists $\delta>0$ such that for any $s \in \mathcal{C}^{0, \alpha}\left(\bar{\Omega} ; \mathbb{R}^{d \times d}\right)$ with $\|s\|_{\mathcal{C}^{0, \alpha}\left(\bar{\Omega} ; \mathbb{R}^{d \times d}\right)} \leq \delta$ we can choose a finite $K \subseteq K_{a d} \backslash \Sigma$ such that

$$
K \times\left\{1, x_{1}, x_{2}\right\}
$$

is a proper set of measurements in $\Omega$ for $a=a_{0}+s$. 
FIgURE 1. A particular combination of the coefficients.
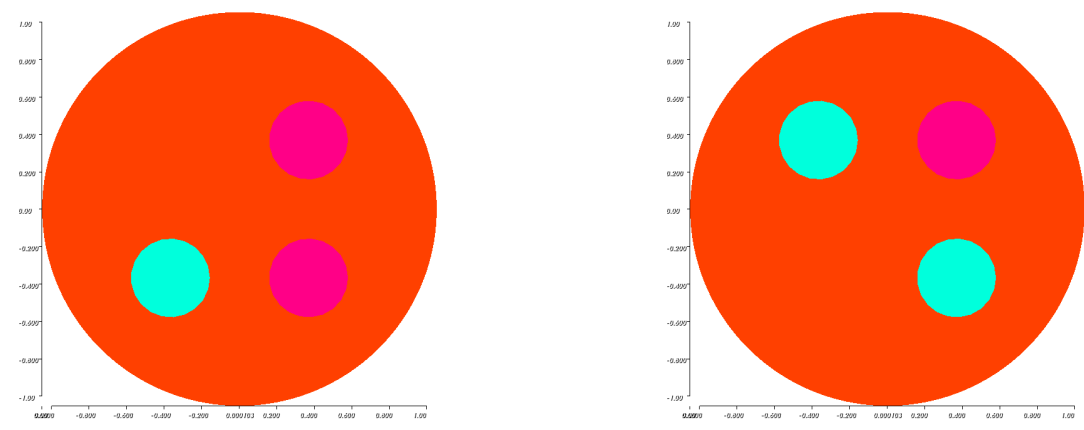

4.5. Numerical Experiments on the Number of Needed Frequencies. The theory developed so far gives conditions on the illuminations to construct complete and proper sets of measurements, provided a finite number of frequencies are chosen in a fixed range. However, we do not provide an estimate on the number of the required frequencies, i.e. the cardinality $\# K$ of $K$. In order to document this point and see what we can expect, we have performed a numerical test in $3^{8}=6561$ different cases, namely for different choices of the parameter distributions in the material. For simplicity, we have decided to consider the two-dimensional case and the concept of proper sets of measurements.

Take $\Omega=B(0,1)$. We now describe the coefficients we have used. Set $c=0.35, r=0.2$ and $P_{1}=(-c,-c), P_{2}=(-c, c), P_{3}=(c,-c)$ and $P_{4}=(c, c)$. Let $\chi_{i}$ be the characteristic function of $B\left(P_{i}, r\right)$ (or, more precisely, a smooth approximation of it). Then we set

$$
a=1+\sum_{i=1}^{4} \alpha_{i} \chi_{i}, \quad q=1+\sum_{i=1}^{4} \beta_{i} \chi_{i}
$$

where $\alpha_{i}, \beta_{i} \in\{0,1,2\}$ (see Figure 1). This construction gives $3^{8}$ different combinations.

In view of Theorem 4.13, we choose the illuminations $\varphi_{1}=1, \varphi_{2}=x_{1}$ and $\varphi_{3}=x_{2}$. The sequence $\left(k_{l}\right)$ introduced in Lemma 4.1 is given by $k_{l}=\lambda_{0}+a+\frac{b}{l+1}$, for some $a, b>0$ such that $\lambda_{0}+a+b<\lambda_{1}$. (As in Proposition 3.1, the sequence $\left(\lambda_{i}\right)$ denotes the Dirichlet eigenvalues of the problem.) These conditions ensure that the chosen frequencies lie between the first and the second Dirichlet eigenvalue, which simplifies the numerical computation. For any combination of the coefficients, we compute the minimum number $\# K$ of needed frequencies such that $\left\{k_{l}: l=\right.$ $0, \ldots, \# K-1\} \times\left\{\varphi_{i}\right\}$ is a proper set of measurements in $\Omega$. The results are summarized in Table 1 . These figures suggest that in practical applications the number of needed frequencies could be quite small.

TABLE 1. Number of combinations of coefficients per number of needed frequencies to obtain a proper set of measurements in $\Omega$.

\begin{tabular}{c|ccc} 
Needed frequencies $(\# K)$ & 2 & 3 & $\geq 4$ \\
\hline Combinations of coefficients & 1609 & 4952 & 0
\end{tabular}




\section{Applications to Microwave Imaging by Ultrasound Deformation}

This section is devoted to the discussion of the hybrid problem introduced in Subsection 2.2 and to the proofs of the results therein presented.

5.1. Formulation of the Problem. The object under examination is a smooth bounded domain $\Omega \subseteq \mathbb{R}^{d}$, for $d=2$ or $d=3$. In the microwave regime, the electric field $u_{k}^{\varphi}$ in $\Omega$ is assumed to satisfy the Helmholtz equation

$$
\left\{\begin{array}{l}
-\operatorname{div}\left(a \nabla u_{k}^{\varphi}\right)-k q u_{k}^{\varphi}=0 \quad \text { in } \Omega \\
u_{k}^{\varphi}=\varphi \quad \text { on } \partial \Omega,
\end{array}\right.
$$

where $a \in \mathcal{C}^{0, \alpha}(\bar{\Omega})$ is the inverse of the magnetic permeability, $q \in L^{\infty}(\Omega)$ is the electric permittivity and $\sqrt{k}$ is the wavenumber (with an abuse of language we shall sometimes refer to $k$ as the frequency or the wavenumber). We assume (4) and (5). The use of the Helmholtz equation is a very common scalar approximation of the Maxwell equations [21, 32, 7, 13. As we have seen in Section 3, problem (26) is well-posed provided that $k$ is not a resonant frequency.

Practitioners can choose a frequency $k>0$ in a fixed range (not a resonant frequency), a real illumination $\varphi$ on the boundary and measure the generated current on the boundary $a \frac{\partial u_{k}^{\varphi}}{\partial \nu}$. As described in [7, these measurements are combined with localized ultrasonic waves focusing in small regions $\omega$ inside $\Omega$. We assume that the electromagnetic parameters are affected linearly with respect to the amplitude of the ultrasonic perturbation, that is supposed to be small. Moreover, this modification is localized only in the region $\omega$. Under these assumptions, denoting the modified coefficients by $\bar{a}$ and $\bar{q}$ we have

$$
\left\{\begin{array}{l}
\bar{a}=a\left(1+c_{a} \alpha \chi_{\omega}\right), \\
\bar{q}=q\left(1+c_{q} \alpha \chi_{\omega}\right),
\end{array}\right.
$$

where $\alpha$ is the amplitude of the ultrasonic signal and $c_{a}$ and $c_{q}$ are known functions. The corresponding electric field $\bar{u}_{k}^{\varphi}$ is the solution to

$$
\left\{\begin{array}{l}
-\operatorname{div}\left(\bar{a} \nabla \bar{u}_{k}^{\varphi}\right)-k \bar{q} \bar{u}_{k}^{\varphi}=0 \quad \text { in } \Omega \\
\bar{u}_{k}^{\varphi}=\varphi \quad \text { on } \partial \Omega .
\end{array}\right.
$$

The density current $a \frac{\partial \bar{u}_{k}^{\varphi}}{\partial \nu}$ on the boundary of the domain is a measurable datum.

We now see how the internal energies can be determined by studying the change between $a \frac{\partial u_{k}^{\varphi}}{\partial \nu}$ and $a \frac{\partial \bar{u}_{k}^{\varphi}}{\partial \nu}$. We consider a wavenumber $k$ and two fixed illuminations $\varphi$ and $\psi$. Suppose the domain $\omega$ to be a small ball inside $\Omega$ of centre $z$. By asymptotic analysis techniques [11, 7], there holds

$$
\begin{aligned}
\int_{\partial \Omega} a\left(\frac{\partial \bar{u}_{k}^{\varphi}}{\partial \nu}\right. & \left.-\frac{\partial u_{k}^{\varphi}}{\partial \nu}\right) \psi d \sigma \\
& =|\omega| \frac{d c_{a}(z) \alpha}{c_{a}(z) \alpha+d} a(z) \nabla u_{k}^{\varphi}(z) \cdot \nabla u_{k}^{\psi}(z)+|\omega| k c_{q}(z) \alpha q(z) u_{k}^{\varphi}(z) u_{k}^{\psi}(z)+o(|\omega|),
\end{aligned}
$$

where $|\omega|$ denotes the Lebesgue measure of $\omega$ and $d \sigma$ the integration with respect to the surface area. Since the left hand side is known, by choosing different values for $\alpha$ the internal power density data

$$
E_{k}^{\varphi \psi}(z)=a(z) \nabla u_{k}^{\varphi}(z) \cdot \nabla u_{k}^{\psi}(z), \quad e_{k}^{\varphi \psi}(z)=q(z) u_{k}^{\varphi}(z) u_{k}^{\psi}(z)
$$


are recovered for every $z \in \Omega^{\prime}$, where $\Omega^{\prime} \Subset \Omega$ is the set of all possible centres where the ultrasonic beams are focused. Note that the "polarized" data with $\varphi \neq \psi$ is available for a fixed $k$, but this argument does not allow the reconstruction of the cross-frequency data

$$
E_{k l}^{\varphi \psi}(z)=a(z) \nabla u_{k}^{\varphi}(z) \cdot \nabla u_{l}^{\psi}(z), \quad e_{k l}^{\varphi \psi}(z)=q(z) u_{k}^{\varphi}(z) u_{l}^{\psi}(z) .
$$

We thus chose not to use cross-frequency data for the reconstruction, in contrast to [7].

Let us now represent the formulation of Problem 2.4. Given a set of measurement $K \times\left\{\varphi_{i}\right\}$ we consider the unique solution $u_{k}^{i} \in H^{1}(\Omega)$ to the problem

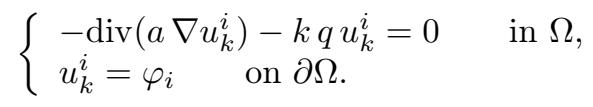

We define the internal data by

$$
e_{k l}^{i j}=q u_{k}^{i} u_{l}^{j}, \quad E_{k l}^{i j}=a \nabla u_{k}^{i} \cdot \nabla u_{l}^{j} .
$$

For simplicity, we denote $e_{k l}=\left(e_{k l}^{i j}\right)_{i j}$ and $e_{k}:=e_{k k}$ and similarly for $E$. The matrices $e_{k}$ and $E_{k}$ are to be considered as known matrix-valued functions. We study the inverse problem of determining the parameters $a$ and $q$ in $\Omega^{\prime}$ from the knowledge in $\Omega^{\prime}$ of $e_{k}$ and $E_{k}$ with a properly chosen set of measurements. Note that this reconstruction problem is slightly different to the one studied in [7], where the full matrices

$$
e=\left[\begin{array}{ccc}
e_{k_{1} k_{1}} & \cdots & e_{k_{1} k_{M}} \\
\vdots & \ddots & \vdots \\
e_{k_{M} k_{1}} & \cdots & e_{k_{M} k_{M}}
\end{array}\right], \quad E=\left[\begin{array}{ccc}
E_{k_{1} k_{1}} & \cdots & E_{k_{1} k_{M}} \\
\vdots & \ddots & \vdots \\
E_{k_{M} k_{1}} & \cdots & E_{k_{M} k_{M}}
\end{array}\right] .
$$

are supposed to be known, with $K=\left\{k_{1}, \ldots, k_{M}\right\}$. In our case, we suppose that only the diagonal blocks are measurable.

5.2. Reconstruction Algorithm. This subsection is devoted to the proofs of the exact formulae given in Subsection 2.2.

5.2.1. Reconstruction Formula for $a / q$. We restate here Theorem 2.6 The new contribution of this work is the proof of the bounds (9).

Theorem 2.6. Take $p, r>0$ and $K \times\left\{\varphi_{i}\right\} \in \mathcal{P}\left(\Omega^{\prime} ; p, r\right)$. Suppose that

$$
\text { (8) }\left|E_{k}^{i i}(x)\right|,\left|e_{k}^{i i}(x)\right| \leq b, \quad x \in \Omega^{\prime} \text {, }
$$

for some $b>0$. Take $x \in \Omega^{\prime}$ and $\bar{k}$ as in Definition 2.5. Then there exists $C=C\left(\lambda, \beta_{1}, b\right)>0$ such that

(9)

$$
\frac{\operatorname{tr}\left(e_{\bar{k}}\right) \operatorname{tr}\left(E_{\bar{k}}\right)-\operatorname{tr}\left(e_{\bar{k}} E_{\bar{k}}\right)}{\operatorname{tr}\left(e_{\bar{k}}\right)^{2}}(x) \geq C p^{2} r^{4}
$$

and $a / q$ is given in terms of the data by

$$
\left|\nabla\left(e_{\bar{k}} / \operatorname{tr}\left(e_{\bar{k}}\right)\right)\right|_{2}^{2} \frac{a}{q}=2 \frac{\operatorname{tr}\left(e_{\bar{k}}\right) \operatorname{tr}\left(E_{\bar{k}}\right)-\operatorname{tr}\left(e_{\bar{k}} E_{\bar{k}}\right)}{\operatorname{tr}\left(e_{\bar{k}}\right)^{2}} \text { in } x .
$$

Remark 5.1. It is worth noting that in presence of noise the dependence of $\bar{k}$ on $x$ does not constitute a source of instability during the reconstruction performed in (10). Take $0<\varepsilon<\min (p, r)$. Since $K=\left\{k_{m}: m=1, \ldots, M\right\}$ is finite, we define for each $m \in\{1, \ldots, M\}$ the set $G_{m}=\left\{x \in \Omega^{\prime}\right.$ : $\left|u_{k_{m}}^{1}(x)\right|>p-\varepsilon$ and $\left.\left|\nabla u_{k_{m}}^{2}(x)\right|\left|\nabla u_{k_{m}}^{3}(x)\right|\left|\sin \theta_{\nabla u_{k_{m}}^{2}, \nabla u_{k_{m}}^{3}}(x)\right|>r-\varepsilon\right\}$. In view of Proposition 3.3,

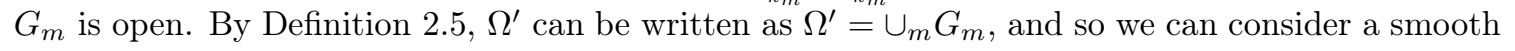


partition of unity $\left\{\psi_{m}\right\}_{m}$ subject to the cover $\left\{G_{m}\right\}_{m}$. Denote by $r_{m}$ the value for $a / q$ obtained via (10) with $\bar{k}=k_{m}$. In view of Theorem 2.6, $r_{m}$ is a well-defined function in $G_{m}$. We can now reconstruct $a / q$ in $\Omega^{\prime}$ using

$$
\frac{a}{q}(x)=\sum_{m=1}^{M} r_{m}(x) \psi_{m}(x), \quad x \in \Omega^{\prime} .
$$

Thus, with this formulation we have removed the dependence of $\bar{k}$ on $x$ and so no additional instability occurs.

Proof. Condition (PSM 1) implies $\operatorname{tr}\left(e_{\bar{k}}\right)>0$ in $\Omega^{\prime}$, and so we may divide by $\operatorname{tr}\left(e_{\bar{k}}\right)$. Following the argument given in the proof of Proposition 3.3 in [7] we obtain

$$
\left|\nabla\left(e_{\bar{k}} / \operatorname{tr}\left(e_{\bar{k}}\right)\right)\right|_{2}^{2} \frac{a}{q}=2 \frac{\operatorname{tr}\left(e_{\bar{k}}\right) \operatorname{tr}\left(E_{\bar{k}}\right)-\operatorname{tr}\left(e_{\bar{k}} E_{\bar{k}}\right)}{\operatorname{tr}\left(e_{\bar{k}}\right)^{2}} .
$$

We now prove (9). For cleanliness of notation we shall denote several positive constants depending on $\lambda$ and $b$ simply by $c=c(\lambda, b)>0$. Until the end of the proof, all the functions will be considered evaluated in $x$. We equip the space of real symmetric matrices with the Hilbert-Schmidt scalar product defined by $\langle A, B\rangle=\operatorname{tr}(A B)$. We claim that

$$
\left|\left(E_{\bar{k}}^{2,3}\right)^{2}-E_{\bar{k}}^{2,2} E_{\bar{k}}^{3,3}\right| \leq c\left\|E_{\bar{k}}\right\|\left(1+\frac{\operatorname{tr}\left(E_{\bar{k}}\right)}{\operatorname{tr}\left(e_{\bar{k}}\right)}\right) \sqrt{1-\cos \theta_{e_{\bar{k}}, E_{\bar{k}}}} .
$$

As a consequence of this inequality, which we shall prove later, we get

$$
\sqrt{1-\cos \theta_{e_{\bar{k}}, E_{\bar{k}}}}\left\|E_{\bar{k}}\right\| \geq c \frac{r^{2},}{1+\frac{\operatorname{tr}\left(E_{\bar{k}}\right)}{\operatorname{tr}\left(e_{\bar{k}}\right)}}
$$

since by ( $(\overline{\mathrm{PSM}} 2)$ there holds

$$
\left|\left(E_{\bar{k}}^{2,3}\right)^{2}-E_{\bar{k}}^{2,2} E_{\bar{k}}^{3,3}\right|=\left.a^{2}\left|\left(\nabla u_{\bar{k}}^{2} \cdot \nabla u_{\bar{k}}^{3}\right)^{2}-\right| \nabla u_{\bar{k}}^{2}\right|^{2}\left|\nabla u_{\bar{k}}^{3}\right|^{2} \mid \geq c r^{2} .
$$

Note that, from the definition of $e_{k}$ and $E_{k}$, we have $\left(e_{\bar{k}}^{i j}\right)^{2}=e_{\bar{k}}^{i i} e_{\bar{k}}^{j j}$ and $\left(E_{\bar{k}}^{i j}\right)^{2} \leq E_{\bar{k}}^{i i} E_{\bar{k}}^{j j}$. In particular,

$$
\left\|e_{\bar{k}}\right\|=\sqrt{\sum_{i, j}\left(e_{\bar{k}}^{i j}\right)^{2}}=\operatorname{tr}\left(e_{\bar{k}}\right), \quad\left\|E_{\bar{k}}\right\|=\sqrt{\sum_{i, j}\left(E_{\bar{k}}^{i j}\right)^{2}} \leq \operatorname{tr}\left(E_{\bar{k}}\right) .
$$

Combining (31) and (32) we obtain

$$
\begin{aligned}
\frac{\operatorname{tr}\left(e_{\bar{k}}\right) \operatorname{tr}\left(E_{\bar{k}}\right)-\operatorname{tr}\left(e_{\bar{k}} E_{\bar{k}}\right)}{\operatorname{tr}\left(e_{\bar{k}}\right)^{2}} & \geq \frac{\operatorname{tr}\left(e_{\bar{k}}\right)\left(1-\cos \theta_{e_{\bar{k}}, E_{\bar{k}}}\right)\left\|E_{\bar{k}}\right\|}{\operatorname{tr}\left(e_{\bar{k}}\right)^{2}} \\
& \geq \frac{c \lambda^{4} r^{4} \operatorname{tr}\left(e_{\bar{k}}\right)}{\operatorname{tr}\left(e_{\bar{k}}\right)^{2} \operatorname{tr}\left(E_{\bar{k}}\right)\left(1+\frac{\operatorname{tr}\left(E_{\bar{k}}\right)}{\operatorname{tr}\left(e_{\bar{k}}\right)}\right)^{2}},
\end{aligned}
$$

which gives the desired result since the denominator is bounded by a constant depending on $b$, and $\operatorname{tr}\left(e_{\bar{k}}\right) \geq \beta_{1} p^{2}$. 
Let us now turn to the proof of (30). We use the notation $g=E_{\bar{k}} /\left\|E_{\bar{k}}\right\|-e_{\bar{k}} /\left\|e_{\bar{k}}\right\|$. By (8) and (32) we have

$$
\begin{aligned}
\left|\left(E_{\bar{k}}^{2,3}\right)^{2}-E_{\bar{k}}^{2,2} E_{\bar{k}}^{3,3}\right| \leq & \left|\left(E_{\bar{k}}^{2,3}\right)^{2}-\frac{\left\|E_{\bar{k}}\right\|^{2}}{\left\|e_{\bar{k}}\right\|^{2}}\left(e_{\bar{k}}^{2,3}\right)^{2}\right|+\left|\frac{\left\|E_{\bar{k}}\right\|^{2}}{\left\|e_{\bar{k}}\right\|^{2}} e_{\bar{k}}^{2,2} e_{\bar{k}}^{3,3}-\frac{\left\|E_{\bar{k}}\right\|}{\left\|e_{\bar{k}}\right\|} e_{\bar{k}}^{2,2} E_{\bar{k}}^{3,3}\right| \\
& +\left|\frac{\left\|E_{\bar{k}}\right\|}{\left\|e_{\bar{k}}\right\|} e_{\bar{k}}^{2,2} E_{\bar{k}}^{3,3}-E_{\bar{k}}^{2,2} E_{\bar{k}}^{3,3}\right| \\
= & \left\|E_{\bar{k}}\right\|\left|E_{\bar{k}}^{2,3}+\frac{\left\|E_{\bar{k}}\right\|}{\left\|e_{\bar{k}}\right\|} e_{\bar{k}}^{2,3}\right|\left|g_{2,3}\right|+\frac{\left\|E_{\bar{k}}\right\|^{2}}{\left\|e_{\bar{k}}\right\|} e_{\bar{k}}^{2,2}\left|g_{3,3}\right| \\
& +\left\|E_{\bar{k}}\right\| E_{\bar{k}}^{3,3}\left|g_{2,2}\right| \\
\leq & c\left\|E_{\bar{k}}\right\|\left(1+\frac{\operatorname{tr}\left(E_{\bar{k}}\right)}{\operatorname{tr}\left(e_{\bar{k}}\right)}\right) \sqrt{1-\cos \theta_{e_{\bar{k}}, E_{\bar{k}}}},
\end{aligned}
$$

since $\left|g_{2,3}\right|+\left|g_{3,3}\right|+\left|g_{2,2}\right| \leq c\|g\| \leq c \sqrt{1-\cos \theta_{e_{\bar{k}}, E_{\bar{k}}}}$.

5.2.2. Reconstruction Formula for $q$. In this subsection we derive the reconstruction formula for $q$ given in Theorem 2.7 It is based on the knowledge of the ratio $G:=a / q$, that can be computed via the formula (10). Since this reconstruction involves the derivative of the data, we need a stable way to obtain $q$ from $G$.

The following lemma reviews the derivatives and the trace of products of functions in Sobolev Spaces.

Lemma 5.2. Take $u, v \in H^{1}(\Omega ; \mathbb{C}) \cap L^{\infty}(\Omega ; \mathbb{C})$. Then $u v \in H^{1}(\Omega ; \mathbb{C}), \nabla(u v)=u \nabla v+v \nabla u$ and $\gamma(u v)=\gamma(u) \gamma(v)$. If in addition $v \geq c>0$ almost everywhere then $u / v \in H^{1}(\Omega ; \mathbb{C}), \nabla(u / v)=$ $\nabla u / v-u \nabla v / v^{2}$ and $\gamma(u / v)=\gamma(u) / \gamma(v)$.

Proof. It easily follows from [23, Section 5.5, Theorem 1] and [24, Lemma 7.5].

We need the following preliminary result, which follows from a straightforward calculation by Lemma 5.2 and Proposition 3.3. The proof is left to the reader.

Lemma 5.3. Let $K \times\left\{\varphi_{i}\right\}$ be a set of measurements. Then $u_{k}^{i} u_{k}^{j} \in H^{1}(\Omega)$ is the unique solution to the problem

$$
\left\{\begin{array}{l}
-\operatorname{div}(a \nabla(u))=2 k e_{k}^{i j}-2 E_{k}^{i j} \quad \text { in } \Omega, \\
u=\varphi_{i} \varphi_{j} \quad \text { on } \partial \Omega,
\end{array}\right.
$$

for every $k, i, j$.

We are now in a position to prove the reconstruction formula for $q$ given in Theorem 2.7 Recall that if we consider a proper set of measurements $K \times\left\{\varphi_{i}\right\}$ we have that

$$
\operatorname{tr}(e)=\sum_{k, i} e_{k}^{i i} \geq c>0 \quad \text { in } \Omega^{\prime}
$$

for some $c>0$.

Theorem 2.7, Let $K \times\left\{\varphi_{i}\right\}$ be a proper set of measurements. Suppose $q \in H^{1}(\Omega)$. Then $\log q$ is the unique solution to the problem

$$
\left\{\begin{array}{l}
-\operatorname{div}(G \operatorname{tr}(e) \nabla u)=-\operatorname{div}(G \nabla(\operatorname{tr}(e)))+2 \sum_{k, i}\left(E_{k}^{i i}-k e_{k}^{i i}\right) \quad \text { in } \Omega^{\prime}, \\
u=\log q_{\mid \partial \Omega^{\prime}} \quad \text { on } \partial \Omega^{\prime} .
\end{array}\right.
$$


Proof. By Proposition 3.3 we infer that $u_{k}^{i}, u_{k}^{j} \in H^{1}(\Omega) \cap L^{\infty}(\Omega)$. Therefore, by Lemma 5.2 we get that $u_{k}^{i} u_{k}^{j} \in H^{1}(\Omega) \cap L^{\infty}(\Omega)$. Since $q \in H^{1}(\Omega) \cap L^{\infty}(\Omega)$ we obtain that $e_{k}^{i j} \in H^{1}(\Omega)$. Hence by Lemma 7.5 in 24] we have

$$
\nabla\left(u_{k}^{i} u_{k}^{j}\right)=\nabla\left(e_{k}^{i j} / q\right)=\left(\nabla e_{k}^{i j}-e_{k}^{i j} \nabla(\log q)\right) / q
$$

with $\log q \in H^{1}(\Omega)$. As a result, in view of Lemma 5.3 it is immediate to show that for every $v \in \mathcal{D}(\Omega)$

$$
\left(2 k e_{k}^{i j}-2 E_{k}^{i j}\right)(v)=-\operatorname{div}\left(G \nabla e_{k}^{i j}\right)(v)+\operatorname{div}\left(G e_{k}^{i j} \nabla(\log q)\right)(v)
$$

whence the result follows by summing all these equations with $i=j$.

5.3. Numerical Experiments. In this section we shall do some numerical simulations of the reconstruction algorithm discussed in the last section. FreeFem ++ has been used. The exact formulae given in Theorem 2.6 and Theorem 2.7 will be used to image the electromagnetic parameters $a$ and $q$.

In both cases, the construction of proper sets of measurements by means of the multi-frequency approach turns out to be effective. Further, in two dimensions, the reconstruction procedure gives better results than the one described in [7! with about one seventh of the available data, the reconstruction errors are about half of the previous ones.

5.3.1. Two-Dimensional Example. Since the two-dimensional case has been tested thoroughly in [7, we have decided to study the same example in order to be able to make a comparison. There are two main differences. First, the formula for the reconstruction of $q$ is not the same. Second, in our case the available data is smaller since we do not use the cross-frequency data.

Let $\Omega=B(0,1)$ be the unit disk. We use a uniform mesh of the disk with about 3000 triangles and 1600 vertices. The coefficients are given by

$$
a=\left\{\begin{array}{ll}
2 & \text { in } B, \\
1.2 & \text { in } C, \\
2.5 & \text { in } E, \\
1 & \text { otherwise }
\end{array} \quad q= \begin{cases}2 & \text { in } B \\
1.8 & \text { in } C \\
1.2 & \text { in } E \\
1 & \text { otherwise }\end{cases}\right.
$$

The set $B$ is the rectangle with diagonal $(0,0.4)-(0.3,0.5)$. The set $C$ is the area delimited by the curve $t \mapsto(0.3+\rho(t) \cos (t),-0.2+\rho(t) \sin (t))$, where $\rho(t)=(20+3 \sin (5 t)-2 \sin (15 t)+\sin (25 t)) / 100$. The set $E$ is the ellipse of centre $(-0.3,0.1)$, with vertical axis of length 0.3 and horizontal axis of length 0.2. These parameters represent three inclusions of different contrast in a homogeneous background medium. This is the typical practical situation, since cancerous tissues have typically higher values in the parameters [34. The coefficients $a$ and $q$ are shown in Figure 2.

Let $\Omega^{\prime}=B(0,0.8)$ be the subdomain where the internal energies are constructed (see Section 5.1). We also suppose that $a$ and $q$ are known in $\Omega \backslash \Omega^{\prime}$. In view of Theorem 4.13, we choose the illuminations $x_{1}+2$ and $x_{2}+2$ and $K=\{1,3,7\}$. In [7], the boundary conditions $1, x_{1}, x_{2}$ and the same $K$ were chosen: these illuminations satisfy the hypotheses of Theorem 4.13 .

Let $a^{*}$ and $q^{*}$ denote the approximated coefficients. We first reconstruct $G=a^{*} / q^{*}$ in $x \in \Omega^{\prime}$ by means of the formula (10)

$$
\frac{a^{*}}{q^{*}}(x)=2 \frac{\operatorname{tr}\left(e_{k}\right) \operatorname{tr}\left(E_{k}\right)-\operatorname{tr}\left(e_{k} E_{k}\right)}{\left|\nabla\left(e_{k} / \operatorname{tr}\left(e_{k}\right)\right)\right|_{2}^{2} \operatorname{tr}\left(e_{k}\right)^{2}}(x)
$$


FiguRE 2. The reference parameters.

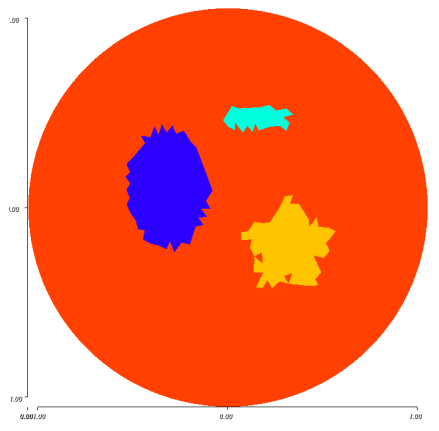

(A) The coefficient $a$.

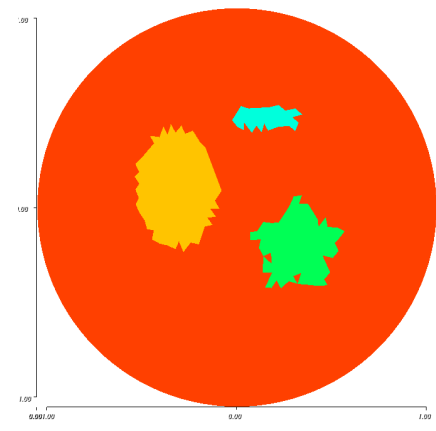

(в) The coefficient $q$.

FiguRE 3. The reconstructed parameters.

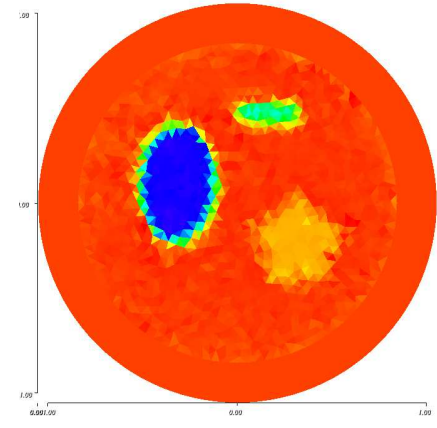

(A) The coefficient $a^{*}$.

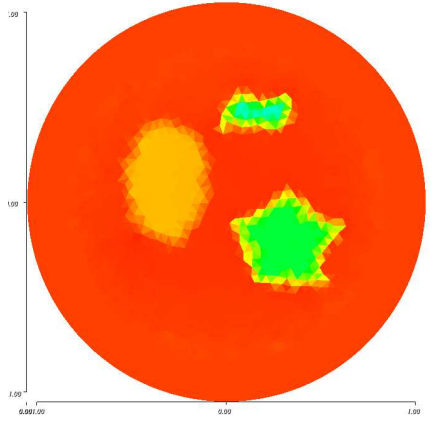

(в) The coefficient $q^{*}$.

averaging over all the $k \in K$ such that the denominator of the right hand side is bigger than $10^{-2}$. Since for every $x \in \Omega^{\prime}$ the set of such $k$ is not empty, the chosen set of measurements turns out to be proper in $\Omega^{\prime}$.

Then, we use Theorem 2.7 to image $\log q^{*} \in H_{0}^{1}\left(\Omega^{\prime}\right)$ :

$$
-\operatorname{div}\left(G \operatorname{tr}(e) \nabla\left(\log q^{*}\right)\right)=-\operatorname{div}(G \nabla(\operatorname{tr}(e)))+2 \sum_{k \in K} \sum_{i=1}^{2}\left(E_{k}^{i i}-k e_{k}^{i i}\right) \quad \text { in } \Omega^{\prime} .
$$

Finally, $a^{*}$ is given by $a^{*}=G q^{*}$, which, in absence of noise, gives a good approximation. The reconstructed coefficients are shown in Figure 3

In Table 2 we compare these findings with the numerical experiments performed in [7]. Even if the non-availability of the cross-frequency data makes the number of measurable internal energies much smaller (about one seventh), the $L^{2}$ norms of the errors $a-a^{*}$ and $q-q^{*}$ in this work are about half the corresponding norms obtained in [7]. 
TABLE 2. A comparison between the numerical experiments carried out in [7] and in this work.

\begin{tabular}{|c|c|c|}
\cline { 2 - 3 } \multicolumn{1}{c|}{} & $\begin{array}{c}\text { Numerical experiments } \\
\text { in [7] }\end{array}$ & $\begin{array}{c}\text { Numerical experiments } \\
\text { in this work }\end{array}$ \\
\hline Illuminations & $1, x_{1}, x_{2}$ & $x_{1}+2, x_{2}+2$ \\
\hline Frequencies $K$ & $1,3,7$ & $1,3,7$ \\
\hline Number of energies & 81 & 12 \\
\hline$\left\|a-a^{*}\right\|_{2}$ & $3.5 \cdot 10^{-1}$ & $1.6 \cdot 10^{-1}$ \\
\hline$\left\|q-q^{*}\right\|_{2}$ & $1.5 \cdot 10^{-1}$ & $0.8 \cdot 10^{-1}$ \\
\hline
\end{tabular}

FigURE 4. A section of the electric permittivity $q$ in $\Omega^{\prime}$.

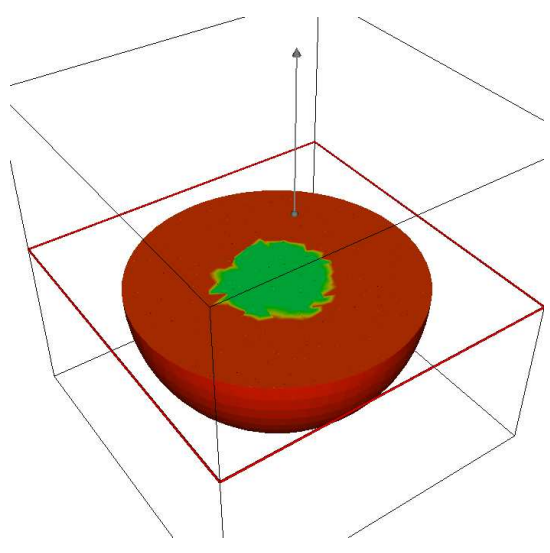

(A) The reference parameter.

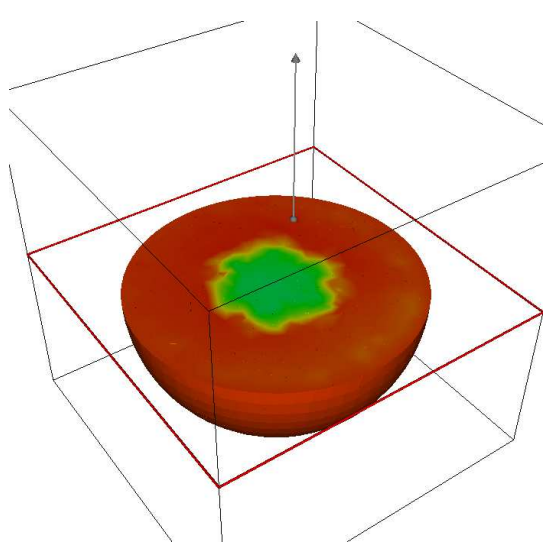

(в) The reconstructed parameter.

5.3.2. Three-Dimensional Example. Take $\Omega=B(0,1)$ and $\Omega^{\prime}=B(0,0.8)$. We use a mesh with about 30000 tetrahedra and 6000 vertices. For simplicity, we choose $a=1$ and $q=1+0.8 \chi_{B(0,0.3)}$ and are interested in imaging the electric permittivity $q$. In view of Theorem 4.14, we choose the set of measurements $\{1,3,7\} \times\left\{x_{1}+2, x_{2}+2\right\}$, which a posteriori turns out to be proper in $\Omega^{\prime}$. The same reconstruction procedure described in the two-dimensional example is used. The reconstruction error is $\left\|q-q^{*}\right\|_{2} \approx 1.3 \cdot 10^{-1}$. A comparison between the reference and the reconstructed parameters is shown in Figure 4

\section{ACKNOWLEDGMENTS}

This work has been done during my D.Phil. at the Oxford Centre for Nonlinear PDE under the supervision of Yves Capdeboscq, which I would like to thank for many stimulating discussions on the topic and for a thorough revision of the paper. I am supported by an EPSRC Research Studentship and by the EPSRC Science \& Innovation Award to the Oxford Centre for Nonlinear $\mathrm{PDE}(\mathrm{EP} / \mathrm{EO} 35027 / 1)$. I would like to thank the referees, whose comments and suggestions greatly improved the quality of this paper. 


\section{REFERENCES}

[1] S. Acosta, S. Chow, J. Taylor, and V. Villamizar. On the multi-frequency inverse source problem in heterogeneous media. Inverse Problems, 28(7):075013, 16, 2012.

[2] G. Alessandrini. An identification problem for an elliptic equation in two variables. Ann. Mat. Pura Appl. (4), 145:265-295, 1986.

[3] G. Alessandrini and R. Magnanini. Elliptic equations in divergence form, geometric critical points of solutions, and Stekloff eigenfunctions. SIAM J. Math. Anal., 25(5):1259-1268, 1994.

[4] H. Ammari. An introduction to mathematics of emerging biomedical imaging, volume 62 of Mathématiques 8 Applications (Berlin) [Mathematics \& Applications]. Springer, Berlin, 2008.

[5] H. Ammari, E. Bonnetier, Y. Capdeboscq, M. Tanter, and M. Fink. Electrical impedance tomography by elastic deformation. SIAM J. Appl. Math., 68(6):1557-1573, 2008.

[6] H. Ammari, E. Bossy, J. Garnier, and L. Seppecher. Acousto-electromagnetic Tomography. SIAM J. Appl. Math., to appear, 2012.

[7] H. Ammari, Y. Capdeboscq, F. de Gournay, A. Rozanova-Pierrat, and F. Triki. Microwave imaging by elastic deformation. SIAM J. Appl. Math., 71(6):2112-2130, 2011.

[8] H. Ammari, P. Garapon, H. Kang, and H. Lee. A method of biological tissues elasticity reconstruction using magnetic resonance elastography measurements. Quart. Appl. Math., 66(1):139-175, 2008.

[9] H. Ammari, J. Garnier, and W. Jing. Resolution and stability analysis in acousto-electric imaging. Inverse Problems, 28(8):084005, 20, 2012.

[10] H. Ammari, J. Garnier, W. Jing, and L. H. Nguyen. Quantitative thermo-acoustic imaging: An exact reconstruction formula. J. Differential Equations, 254(3):1375-1395, 2013.

[11] H. Ammari and H. Kang. Polarization and moment tensors, volume 162 of Applied Mathematical Sciences. Springer, New York, 2007. With applications to inverse problems and effective medium theory.

[12] H. Ammari and H. Kang. Expansion methods. In Handbook of Mathematical Methods in Imaging, pages 447-499. Springer, New York, 2011.

[13] G. Bal. Hybrid inverse problems and internal functionals. In G. Uhlmann, editor, Inverse Problems and Applications: Inside Out II, volume 60 of Math. Sci. Res. Inst. Publ., pages 325-368. Cambridge Univ. Press, Cambridge, 2013.

[14] G. Bal, E. Bonnetier, F. Monard, and F. Triki. Inverse diffusion from knowledge of power densities. Arxiv preprint arXiv:1110.4577, 2011.

[15] G. Bal, K. Ren, G. Uhlmann, and T. Zhou. Quantitative thermo-acoustics and related problems. Inverse Problems, 27(5):055007, 15, 2011.

[16] G. Bal and G. Uhlmann. Inverse diffusion theory of photoacoustics. Inverse Problems, 26(8):085010, $20,2010$.

[17] G. Bal and G. Uhlmann. Reconstruction of coefficients in scalar second-order elliptic equations from knowledge of their solutions. arXiv preprint arXiv:1111.5051, 2011.

[18] G. Bao, J. Lin, and F. Triki. A multi-frequency inverse source problem. J. Differential Equations, 249(12):3443$3465,2010$.

[19] P. Bauman, A. Marini, and V. Nesi. Univalent solutions of an elliptic system of partial differential equations arising in homogenization. Indiana University Mathematics Journal, 50(2):747-758, 2001.

[20] M. Briane, G. W. Milton, and V. Nesi. Change of sign of the corrector's determinant for homogenization in three-dimensional conductivity. Arch. Ration. Mech. Anal., 173(1):133-150, 2004.

[21] A. E. Bulyshev, A. E. Souvorov, S. Y. Semenov, R. H. Svenson, A. G. Nazarov, Y. E. Sizov, and G. P. Tatsis. Three-dimensional microwave tomography. Theory and computer experiments in scalar approximation. Inverse Problems, 16(3):863-875, 2000.

[22] Y. Capdeboscq, J. Fehrenbach, F. de Gournay, and O. Kavian. Imaging by modification: numerical reconstruction of local conductivities from corresponding power density measurements. SIAM J. Imaging Sci., 2(4):10031030, 2009.

[23] L. C. Evans. Partial differential equations, volume 19 of Graduate Studies in Mathematics. American Mathematical Society, Providence, RI, 1998.

[24] D. Gilbarg and N. S. Trudinger. Elliptic partial differential equations of second order. Classics in Mathematics. Springer-Verlag, Berlin, 2001. Reprint of the 1998 edition.

[25] O. Kavian. Lectures on parameter identification. In Three courses on partial differential equations, volume 4 of IRMA Lect. Math. Theor. Phys., pages 125-162. de Gruyter, Berlin, 2003. 
[26] P. Kuchment. Mathematics of hybrid imaging: A brief review. In Irene Sabadini and Daniele C Struppa, editors, The Mathematical Legacy of Leon Ehrenpreis, volume 16 of Springer Proceedings in Mathematics, pages 183208. Springer Milan, 2012.

[27] Tae Hwi Lee, Chi Young Ahn, Oh In Kwon, and Jin Keun Seo. A hybrid one-step inversion method for shear modulus imaging using time-harmonic vibrations. Inverse Problems, 26(8):085014, 2010.

[28] F. Monard and G. Bal. Inverse anisotropic diffusion from power density measurements in two dimensions. Inverse Problems, 28(8):084001, 20, 2012.

[29] M. Renardy and R. C. Rogers. An introduction to partial differential equations, volume 13 of Texts in Applied Mathematics. Springer-Verlag, New York, second edition, 2004.

[30] J. Sylvester and G. Uhlmann. A global uniqueness theorem for an inverse boundary value problem. Ann. of Math. (2), 125(1):153-169, 1987.

[31] F. Triki. Uniqueness and stability for the inverse medium problem with internal data. Inverse Problems, 26(9):095014, 11, 2010.

[32] M. S. Vogelius and D. Volkov. Asymptotic formulas for perturbations in the electromagnetic fields due to the presence of inhomogeneities of small diameter. M2AN Math. Model. Numer. Anal., 34(4):723-748, 2000.

[33] E. F. Whittlesey. Analytic functions in Banach spaces. Proc. Amer. Math. Soc., 16:1077-1083, 1965.

[34] T. Widlak and O. Scherzer. Hybrid tomography for conductivity imaging. Inverse Problems, 28(8):084008, 28, 2012.

Mathematical Institute, University of Oxford, Andrew Wiles Building, Radcliffe Observatory Quarter, Woodstock Road, Oxford OX2 6GG, UK.

E-mail address: giovanni.alberti@maths.ox.ac.uk 